\title{
Studying the Implications of Consumption and Asset Return Data for Stochastic Discount Factors in Incomplete International Economies
}

\author{
Gurdip Bakshi ${ }^{1}$, Mario Cerrato ${ }^{2}$, and John Crosby $^{3}$ \\ ${ }^{1}$ University of Maryland, gbakshi@rhsmith.umd.edu \\ ${ }^{2}$ Glasgow University, mario.cerrato@glasgow.ac.uk \\ ${ }^{3}$ University of Technology, Sydney Business School, johnc2205@yahoo.com
}

First Draft, August 2014

October 25, 2016

\begin{abstract}
We develop an incomplete markets framework to synthesize domestic and foreign stochastic discount factors (SDFs) that are consistent with limited international risk sharing. The fundamental departure in our paper is that exchange rate growth need not equal the ratio of SDFs, and we develop a restriction that precludes "good deals" in international economies with incomplete markets. Our innovation is to study an incomplete markets problem that is consistent with SDFs that (i) are nonnegative, (ii) correctly price returns, and (iii) disallow "good deals."
\end{abstract}

Keywords: Incomplete markets, stochastic discount factors, unspanned components, limited risk sharing

This is a substantially revised version of our paper previously circulated as "Risk Sharing in International Economies and Market Incompleteness" (SSRN: http://ssrn.com/abstract=2480679). We are completely in debt of John Cochrane for his many detailed comments that have improved our paper. We thank Michael Brandt, Craig Burnside, Ric Colacito, Ales Cerny, Fousseni Chabi-Yo, Bernard Dumas, Xiaohui Gao, Lorenzo Garlappi, Nicolae Garleanu, Tarek Hassan, Steve Heston, Pete Kyle, Mark Loewenstein, Anthony Neuberger, Anna Pavlova, Lasse Pedersen, Ilaria Piatti, Xiao Qiao, Tarun Ramadorai, Robert Ready, Helene Rey, Shrihari Santosh, Alexander Schiller, Alexis Akira Toda, Laura Veldkamp, Liuren Wu, and Jinming Xue for helpful conversations and feedback, as well as seminar and conference participants at the 27th Australasian Finance and Banking Conference (December 2014), University of Maryland, University of New South Wales Business School, University of Queensland Business School, SIRE Conference at the Adam Smith Business School, University of Sydney Business School, Victoria University of Wellington, Warwick Business School, University College Dublin School of Business, IF2015 Annual Conference in International Finance (Copenhagen Business School), and 2016 American Finance Association meetings (San Francisco). We welcome comments, including references to related papers that we have inadvertently overlooked. Any errors are our responsibility alone. The computer codes are available from any of the authors. 


\section{Introduction}

This paper proposes a framework for characterizing SDF pairs in incomplete international economies. Instrumental to our developments are three features. First, the analysis is anchored to limited consumption risk sharing. Second, one can accommodate a relatively large difference between the unspanned components of the SDFs. Third, we can plausibly model the volatility of SDFs and their correlations. Our new angle of incomplete markets and the depiction of the unspanned components of the SDFs are relevant to the mechanism by which risks are shared - or not shared across international borders, potentially allowing us to shed light on the workings of international finance puzzles.

How high are the correlations between SDFs in international economies? The bottom line view is spearheaded by Brandt, Cochrane, and Santa-Clara (2006), who feature economies in which $m_{t+1}\left(\frac{S_{t+1}}{S_{t}}\right)-m_{t+1}^{\star}=0\left(m_{t+1}\right.$ (respectively, $\left.m_{t+1}^{\star}\right)$ is the domestic (foreign) SDF, and $\frac{S_{t+1}}{S_{t}}$ is the exchange rate growth with the foreign currency as the reference) and argue that correlations must be high. In a potential paradigm shift, we propose an incomplete markets setting in which there is an infinite number of $m_{t+1}$ and $m_{t+1}^{\star}$ pairs that may or may not satisfy $m_{t+1}\left(\frac{S_{t+1}}{S_{t}}\right)-m_{t+1}^{\star}=0$, and show that the correlation between $m_{t+1}$ and $m_{t+1}^{*}$ need not be high, consistent with the empirical evidence on limited consumption-based risk sharing. Related to our motivation to study incomplete markets, an evolving literature has treated the high correlation between $m_{t+1}$ and $m_{t+1}^{*}$ as a salient feature to be modeled theoretically and reconciled empirically within the broader swath of international finance puzzles.

What do we do differently? First, we consider a discrete-time economy with the understanding that there is an infinite number of SDFs in incomplete markets. Second, unlike most others, we do not assume that $m_{t+1}\left(\frac{S_{t+1}}{S_{t}}\right)-m_{t+1}^{\star}=0$, and we develop a restriction that precludes "good deals" in international economies with incomplete markets. Specifically, a good deal is the possibility of forming a portfolio, which has an implausibly high reward-for-risk. We show that ruling out an implausibly high reward-for-risk in an international economy places an upper bound on the dispersion of SDFs. Third, we develop a new theoretical optimization problem (with a quadratic objective and both quadratic and linear constraints) that solves for the lowest SDF covariances in 
the space of the two SDFs subject to the constraint that the two SDFs preclude good deals, the SDFs price the returns on traded assets, and the SDFs be nonnegative.

A core derived feature of our model is an additive form of the SDFs in which the SDFs are analytically decomposed into their spanned and unspanned components, and a low correlation between $m_{t+1}$ and $m_{t+1}^{*}$ can arise when the country-specific unspanned components are negatively correlated. We describe how our theoretical approach offers a distinction from the work of Backus, Foresi, and Telmer (2001) and can be consistent with the data on limited consumption risk sharing.

Three questions are the centerpiece of our research: What are the consequences of incomplete markets for the SDF pairs $\left(m_{t+1}, m_{t+1}^{*}\right)$ in international economies? What are the quantitative attributes of volatilities and correlation between SDFs that are consistent with consumption and asset return data, and consistent with an economically plausible lower bound on covariances? What does our incomplete markets approach say about classic international finance puzzles?

A key input to the theoretical problem and empirical assessment is the market incompleteness parameter $\Theta$ that quantifies an upper bound on the dispersion of the SDFs. The identifiable restriction of incomplete markets and limited risk sharing is the positivity of $\Theta$. We motivate an algorithm to estimate $\Theta$ from consumption growth data, in conjunction with asset return data. The algorithm encapsulates the idea that deviations from complete markets cannot be measured from asset return moments alone. This feature arises not because of choice or theoretical design, but by the nature of the incomplete markets problem in which there is an infinite number of SDFs.

With our new framework for characterizing $\left(m_{t+1}, m_{t+1}^{*}\right)$ pairs, there exists a market incompleteness parameter $\Theta$ for which values of international risk sharing, based on asset returns, can be aligned with those from consumption growth, and our empirical analysis is backed by evidence from 10 industrialized countries (45 country pairs) over 40 years. Under our empirical reconciliation, we consider returns of a risk-free bond and an equity index from each half of a country pair, together with the returns of the U.S. 30-year Treasury bond and the world equity index that are common to all country pairs. This amounts to a universe of six asset returns with multi-currency and multi-country exposures. The approach can be tailored to consider expanded (or reduced) sets of assets, and we show that our headline conclusions relating to SDF volatilities and correlations, and the size of the unspanned components, are robust to the number of assets employed. 
Section 3.3 shows that our incomplete markets approach can prove useful in interpreting international finance puzzles. More specifically, the additive form of the SDFs employed in our paper refutes a notion that the degree of market incompleteness affects the expected excess return of foreign exchange (the forward premium puzzle; Fama (1984) and Backus, Foresi, and Telmer (2001)). Additionally, with respect to the Backus and Smith puzzle, we show that a regression of log exchange rate growth on log relative SDFs synthesized under our theoretical approach produces slope coefficients similar to those observed in implementations of the Backus and Smith regressions. Finally, the question of low volatility of exchange rate growth (the volatility/risk sharing puzzle) was addressed in Brandt, Cochrane, and Santa-Clara (2006) by arguing that the correlations between log SDFs must be high. In contrast, our tenet of incomplete markets furnishes relatively low correlations, while producing plausible SDF volatilities and limited risk sharing.

Related Literature: Our work connects to a body of literature that is at the intersection of consumption risk sharing, asset pricing, exchange rates, and incomplete markets. For example, Lewis (1996) finds some supporting empirical evidence for the idea that the documented low consumption growth correlations and, thus, low international risk sharing, may be explainable by incomplete markets. The work of Burnside and Graveline (2014), Corsetti, Dedola, and Leduc (2008), Kim and Schiller (2015), and Zhang (2015) further motivates us to formalize the implications of incomplete markets for limited risk sharing and international puzzles.

While many scholars are beginning to expose the consequences of incomplete markets for puzzles in international finance, the extant approaches are different from ours. In particular, the novelty of our solution is that the unspanned components are analytically determined as a part of our theoretical problem, and their properties are linked to the degree of market incompleteness. Thus, methodologically, our approach is substantively distinct from Pavlova and Rigobon (2007), Maurer and Tran (2015), and Stathopoulos (2015), in which the goal is not to model the properties of the unspanned components of the domestic and foreign SDFs or their correlations.

How does our incomplete markets approach with " $m_{t+1}\left(\frac{S_{t+1}}{S_{t}}\right)-m_{t+1}^{\star} \neq 0$ " compare to the multiplicative wedge approach proposed in Backus, Foresi, and Telmer (2001, equation (8)), and developed further in Lustig and Verdelhan (2016, equations (7) and (8))? Specifically, the latter characterizes the multiplicative wedge perturbation $\eta_{t+1}$ that satisfies $m_{t+1}\left(\frac{S_{t+1}}{S_{t}}\right)=m_{t+1}^{*} e^{\eta_{t+1}}$, in 
conjunction with correct pricing and distributional assumptions about $\eta_{t+1}$ and the SDFs. We defer a formal discussion of the multiplicative wedge approach to Section 2.7. Importantly, a key economic distinction illustrated in our study is that our solution with additive form of the SDFs is outside of the class of SDFs that are supportable through the multiplicative wedge approach.

Our results also stand in contrast to Lustig and Verdelhan (2016, Proposition 1 and Corollary 1 through 3) with respect to (i) the forward premium puzzle, (ii) the Backus and Smith puzzle, and (iii) the volatility/risk sharing puzzle. First, Bakshi and Crosby (2016, Claim 1) also consider the multiplicative wedge approach with incomplete spanning and show that the expected excess return of foreign exchange (i.e., the currency risk premium) is detached from the multiplicative wedge perturbation. This result is obtained in both a model-free and a distribution-free setting, and contradicts Lustig and Verdelhan (2016, Corollary 2 and Corollary 5). Second, the analysis of Bakshi and Crosby (2016, Claim 2) culminates in an additional result that log changes in the exchange rate, and its quadratic variation, are uncorrelated with the multiplicative wedge perturbation. Third, the incomplete spanning approach in Lustig and Verdelhan cannot, and does not, impact the correlation between the SDFs, and can lower the risk sharing index only under stringent restrictions on the distributional properties of the multiplicative wedge perturbation. Our theoretical and empirical analysis in Section 3.3 further distills why the results in Lustig and Verdelhan are conceptually different than those developed by us in the context of the additive form of SDFs.

The studies of Colacito and Croce $(2011,2013)$ consider a two-country asset pricing model with long-run risk and recursive preferences to reconcile the correlation between asset returns and the correlation between consumption growth. Their model, through the mechanism of a high correlation of the long-run components of consumption growth, produces correlated SDFs, while maintaining a low unconditional correlation between the consumption growth of the two countries. Additionally, Favilukis, Garlappi, and Neamati (2015) employ restrictions in financial trade to induce market incompleteness, and show that their framework could explain certain facts, including a positive correlation between currency appreciation and consumption growth. The study of Gabaix and Maggiori (2015) allows for incomplete markets in their work on international trade and exchange rates. We develop a framework in which SDFs are not unique, and an incomplete markets setting is at the center of a restriction that precludes extremely lucrative trading opportunities. 


\section{A framework for analyzing incomplete international economies}

This section formulates a theoretical problem that characterizes a lower bound on the covariance between SDFs in incomplete international economies, subject to correct pricing. The problem is further disciplined by the imposition of a constraint that precludes trading opportunities with unreasonable reward-for-risk. The key departure from others is that we can analytically decompose domestic and foreign SDFs into spanned and unspanned components in such a way that the correlation between the SDFs is lowered in an economically motivated fashion. The solution traits hinge on the properties of the asset return data. We illustrate the relevance of our approach in addressing international finance puzzles, while recognizing that there is a multitude of SDFs in incomplete markets. The developed theoretical links are instrumental to our empirical investigation.

\subsection{The economic environment}

We consider a discrete-time economy with two dates, namely, $t$, the current time, and $t+1$, the time one period ahead. There are $\mathbb{J}$ (finite or infinite) possible states of the world at time $t+1$. We consider two countries, denoted domestic and foreign, and use a superscript ${ }^{\star}$ to denote quantities in the foreign country.

The exchange rate, defined as the number of units of domestic currency per unit of foreign currency, at time $t$, is denoted by $S_{t}$. The foreign currency is the reference. We assume that asset markets are frictionless. For example, there are no bid-ask spreads and no short sale constraints.

We assume that there are $N$ assets that can be traded by both domestic and foreign investors and that none of the assets is redundant. Denote by $\mathbf{R}_{t+1}$ and $\mathbf{R}_{t+1}^{\star}$ the $N$-dimensional vector of domestic and foreign gross returns. Included within the return vectors $\mathbf{R}_{t+1}$ and $\mathbf{R}_{t+1}^{\star}$ are risk-free bonds in the domestic and foreign country with gross return $R_{f}$ and $R_{f}^{\star}$, respectively.

More generally, we include all available asset returns, in either currency, in both $\mathbf{R}_{t+1}$ and $\mathbf{R}_{t+1}^{\star}$. This means that $\mathbf{R}_{t+1}$ and $\mathbf{R}_{t+1}^{\star}$ are related by

$$
\mathbf{R}_{t+1}=\left(S_{t+1} / S_{t}\right) \mathbf{R}_{t+1}^{\star}
$$


Hence, when we refer to domestic and foreign returns, "domestic" and "foreign" refer to the currency in which the return is made - not, for example, to the country in which the equity index is based.

Let $m_{t+1}$ and $m_{t+1}^{\star}$ denote the domestic and foreign SDFs. Importantly, we do not assume complete markets, so $m_{t+1}$ and $m_{t+1}^{\star}$ are not unique.

To rule out extreme counterexamples, assume that the first and second moments of all relevant quantities, for example, $m_{t+1}, m_{t+1}^{\star}, \mathbf{R}_{t+1}$, and $\mathbf{R}_{t+1}^{\star}$, exist and are finite. In particular, $\mathbb{E}_{t}\left[m_{t+1}^{2}\right]<$ $+\infty$ and $\mathbb{E}_{t}\left[\left(m_{t+1}^{\star}\right)^{2}\right]<+\infty$ and, hence, $\left|\mathbb{E}_{t}\left[m_{t+1} m_{t+1}^{\star}\right]\right|<+\infty$ by the Cauchy-Schwarz inequality, where $\mathbb{E}_{t}[$.$] indicates time t$ conditional expectation. Let $\operatorname{Var}[\cdot]$ and $\operatorname{Cov}[\cdot, \cdot]$ denote variance and covariance, respectively.

\subsection{Implications of incomplete markets}

Since $m_{t+1}$ and $m_{t+1}^{\star}$ price domestic returns $\mathbf{R}_{t+1}$ and foreign returns $\mathbf{R}_{t+1}^{\star}$, respectively,

$$
\mathbb{E}_{t}\left[m_{t+1} \mathbf{R}_{t+1}\right]=\mathbf{1} \quad \text { and } \quad \mathbb{E}_{t}\left[m_{t+1}^{\star} \mathbf{R}_{t+1}^{\star}\right]=\mathbf{1},
$$

where 1 denotes an $N$-dimensional vector of ones. However, since $\mathbf{R}_{t+1}=\left(\frac{S_{t+1}}{S_{t}}\right) \mathbf{R}_{t+1}^{\star}$, we also have $\mathbb{E}_{t}\left[m_{t+1}\left(\frac{S_{t+1}}{S_{t}}\right) \mathbf{R}_{t+1}^{\star}\right]=\mathbf{1}$ and isomorphically $\mathbb{E}_{t}\left[m_{t+1}^{*} \mathbf{R}_{t+1} /\left(\frac{S_{t+1}}{S_{t}}\right)\right]=\mathbf{1}$.

Subtracting $\mathbb{E}_{t}\left[m_{t+1}^{\star} \mathbf{R}_{t+1}^{\star}\right]=\mathbf{1}$ from $\mathbb{E}_{t}\left[m_{t+1}\left(\frac{S_{t+1}}{S_{t}}\right) \mathbf{R}_{t+1}^{\star}\right]=\mathbf{1}$, and $\mathbb{E}_{t}\left[m_{t+1} \mathbf{R}_{t+1}\right]=\mathbf{1}$ from $\mathbb{E}_{t}\left[m_{t+1}^{*} \mathbf{R}_{t+1} /\left(\frac{S_{t+1}}{S_{t}}\right)\right]=\mathbf{1}$ implies:

$$
\mathbb{E}_{t}\left[\left(m_{t+1}\left(\frac{S_{t+1}}{S_{t}}\right)-m_{t+1}^{\star}\right) \mathbf{R}_{t+1}^{\star}\right]=0 \quad \text { and } \quad \mathbb{E}_{t}\left[\left(m_{t+1}-m_{t+1}^{\star} /\left(\frac{S_{t+1}}{S_{t}}\right)\right) \mathbf{R}_{t+1}\right]=0
$$

Since equation (3) is true for all returns $\mathbf{R}_{t+1}^{\star}$ and $\mathbf{R}_{t+1}$, it is true for the foreign risk-free return $R_{f}^{\star}$ and the domestic risk-free return $R_{f}$, which leads to the implications:

$$
\mathbb{E}_{t}\left[m_{t+1}\left(\frac{S_{t+1}}{S_{t}}\right)-m_{t+1}^{\star}\right]=0 \quad \text { and } \quad \mathbb{E}_{t}\left[m_{t+1}-m_{t+1}^{\star} /\left(\frac{S_{t+1}}{S_{t}}\right)\right]=0
$$

Consider the (class of) random variables $\left(\xi_{t+1}, \xi_{t+1}^{*}\right)$, defined by

$$
\xi_{t+1} \equiv m_{t+1}\left(\frac{S_{t+1}}{S_{t}}\right)-m_{t+1}^{\star} \quad \text { and } \quad \xi_{t+1}^{*} \equiv m_{t+1}-m_{t+1}^{\star} /\left(\frac{S_{t+1}}{S_{t}}\right)
$$


Definition. (Incomplete markets problem) By choice of $\left(m_{t+1}, m_{t+1}^{*}\right)$ solve

$$
\begin{array}{ll}
\mathbb{E}_{t}\left[\xi_{t+1}\right]=0, & \mathbb{E}_{t}\left[\xi_{t+1} \mathbf{R}_{t+1}^{\star}\right]=0, \\
\mathbb{E}_{t}\left[\xi_{t+1}^{*}\right]=0, & \mathbb{E}_{t}\left[\xi_{t+1}^{*} \mathbf{R}_{t+1}\right]=0,
\end{array}
$$

where the random variables $\left(\xi_{t+1}, \xi_{t+1}^{*}\right)$ defined in (5) are not unique.

The incomplete markets problem agrees with a view that there are some states for which no Arrow-Debreu security trades. More formally, with $\operatorname{span}(\mathbf{R}) \equiv\left\{\boldsymbol{w}^{\prime} \mathbf{R}: \boldsymbol{w} \in \mathbb{R}^{N}\right\}$ denoting the set of possible portfolio returns, it agrees with $\operatorname{span}(\mathbf{R}) \neq \mathbb{R}^{\mathbb{I}}$, with $N<\mathbb{J}$ (e.g., Duffie (1992, page 8)).

In the case of complete markets, $\left(\xi_{t+1}, \xi_{t+1}^{*}\right)$ satisfying (6) and (7) are unique and identically zero in every state. Correspondingly, there is an Arrow-Debreu security tradeable for every $t+1$ state of the world, which implies, in the absence of arbitrage, that $S_{t} m_{t+1}^{\star}=S_{t+1} m_{t+1}$, or equivalently, we obtain the relation (e.g., Backus, Foresi, and Telmer (2001, Proposition 1, equation (7))),

$$
m_{t+1}\left(\frac{S_{t+1}}{S_{t}}\right)-m_{t+1}^{\star}=0 \text { in a complete markets setting. }
$$

In incomplete markets, some $m_{t+1}$ and $m_{t+1}^{\star}$ satisfy $m_{t+1}\left(\frac{S_{t+1}}{S_{t}}\right)-m_{t+1}^{\star}=0$, and some do not.

The statement that " $m_{t+1}\left(\frac{S_{t+1}}{S_{t}}\right)-m_{t+1}^{\star}$ need not equal zero" is intuitive, because, in incomplete markets, there are some outcomes for which no Arrow-Debreu security trades, and different investors will place different marginal utility on those outcomes. For example, if a representative agent exists in each country and if, say, the domestic agent is more risk averse than the foreign counterpart, then the former will assign greater marginal utility to unpleasant states.

We do stress that equations (3) and (4) always hold, regardless of whether the market is complete or incomplete. Brandt, Cochrane, and Santa-Clara (2006, Section 1.2) highlight the potential significance of incomplete markets, but in the end their analysis features $m_{t+1}\left(\frac{S_{t+1}}{S_{t}}\right)-m_{t+1}^{\star}=0$.

It simplifies the exposition and analytical characterizations if we define (note $S_{t+1} / S_{t}>0$ ) the $N$-dimensional vector $\mathbf{Z}_{t+1}$ by

$$
\mathbf{Z}_{t+1} \equiv \sqrt{S_{t+1} / S_{t}} \mathbf{R}_{t+1}^{\star}=\mathbf{R}_{t+1} / \sqrt{S_{t+1} / S_{t}}
$$


Further, we define

$$
y_{t+1} \equiv m_{t+1} \sqrt{S_{t+1} / S_{t}}, \quad \text { and } \quad y_{t+1}^{\star} \equiv m_{t+1}^{\star} / \sqrt{S_{t+1} / S_{t}},
$$

which implies $y_{t+1} y_{t+1}^{\star}=m_{t+1} m_{t+1}^{\star}$. For later use, observe that $y_{t+1} y_{t+1}^{\star}$ has a bounded expectation, since $\left|\mathbb{E}_{t}\left[y_{t+1} y_{t+1}^{\star}\right]\right|=\left|\mathbb{E}_{t}\left[m_{t+1} m_{t+1}^{\star}\right]\right|<+\infty$.

Furthermore, from equation (2), it must hold that

$$
\mathbb{E}_{t}\left[y_{t+1} \mathbf{Z}_{t+1}\right]=\mathbf{1} \quad \text { and } \quad \mathbb{E}_{t}\left[y_{t+1}^{\star} \mathbf{Z}_{t+1}\right]=\mathbf{1}
$$

Equations (9) and (11) imply that $\mathbf{Z}$ can be interpreted as the gross returns in a hypothetical economy in which the gross returns are the geometric average of $\mathbf{R}$ and $\mathbf{R}^{\star}$ (since (9) implies $\left.\left(\mathbf{R R}^{\star}\right)^{\frac{1}{2}}=\mathbf{Z}\right)$. Further, $y_{t+1}$ and $y_{t+1}^{\star}$ can be interpreted as SDFs in this hypothetical economy.

The transformations in (9) and (10) are merely devices that allow cash flow pricing in a symmetric fashion, circumventing the need to duplicate calculations in different currency units.

If the market were to be complete, equation (8) would imply $y_{t+1}=y_{t+1}^{\star}$. Further, if the market were to be complete, without loss of generality, we can assume that Arrow-Debreu securities, for every time $t+1$ state of the world, trade and then $y_{t+1}=y_{t+1}^{\star}$ is equivalent to saying that domestic investors and foreign investors agree on their prices.

How does our approach with " $y_{t+1}$ not equal to $y_{t+1}^{*}$," or $m_{t+1}\left(\frac{S_{t+1}}{S_{t}}\right)-m_{t+1}^{\star}$ need not equal zero, compare to the multiplicative wedge approach in Backus, Foresi, and Telmer (2001) and Lustig and Verdelhan (2016)? It suffices to say for now that our constructions (i) maintain symmetry, (ii) facilitate an analytical solution that decomposes each SDF into its spanned and unspanned components, and (iii) are tractable for studying the correlation between domestic and foreign SDFs. The crucial takeaway from Section 2.7 is that our developed SDFs are not encompassed within the class of SDFs that are possible with the multiplicative wedge approach.

Whether markets are complete or incomplete, domestic and foreign investors agree on the prices of securities in the linear span of $\mathbf{R}$ ( or $\mathbf{R}^{\star}$ ). The takeaway is that in incomplete markets, $m_{t+1}\left(\frac{S_{t+1}}{S_{t}}\right)-m_{t+1}^{\star}$ need not equal zero, $y_{t+1}$ need not equal $y_{t+1}^{\star}$, and the valuations of domestic and foreign investors need not coincide for securities that are not in the linear span of $\mathbf{R}$ (or $\mathbf{R}^{\star}$ ). 


\subsection{Motivating a constraint on reward-for-risk in the international economy}

How different can those valuations be in incomplete markets? The purpose of this subsection is to derive a restriction of the form $\mathbb{E}_{t}\left[\left(y-y^{\star}\right)^{2}\right] \leq \Theta^{2}$ for some constant $\Theta$, by showing that it is equivalent to placing an economically motivated bound on the differences in valuation of securities that are not in the linear span of $\mathbf{R}\left(\right.$ or $\left.\mathbf{R}^{\star}\right)$.

The substantive content of an upper bound $\mathbb{E}_{t}\left[\left(y-y^{\star}\right)^{2}\right] \leq \Theta^{2}$ arises in incomplete markets when $y$ need not equal $y^{\star}$ and is related to the degree of market incompleteness.

Following Cochrane (2005, pages 94-95), we project $y$ and $y^{\star}$ onto the space of gross returns $\mathbf{Z}$ (omitting time subscripts for brevity):

$$
y=y_{\mathbf{z}}+\frac{1}{2} q_{0} \delta, \quad \text { and } \quad y^{\star}=y_{\mathbf{z}}+\frac{1}{2} q_{0}^{\star} \delta
$$

where $q_{0}$ and $q_{0}^{\star}$ are constant scalars and $y_{\mathbf{z}}$ and $\delta$ are random variables that satisfy

$$
\mathbb{E}_{t}\left[y_{\mathbf{z}} \mathbf{Z}\right]=1, \quad \mathbb{E}_{t}\left[y_{\mathbf{z}} \delta\right]=0, \quad \mathbb{E}_{t}[\delta \mathbf{Z}]=0 \text { for each element of } \mathbf{Z}, \quad \text { and } \quad \mathbb{E}_{t}\left[\delta^{2}\right]=1
$$

The decomposition in equation (12) breaks $y$ and $y^{\star}$ into two components. The first component

$y_{\mathbf{z}}=\mathbf{1}^{\prime}\left(\mathbb{E}_{t}\left[\mathbf{Z Z}^{\prime}\right]\right)^{-1} \mathbf{Z}$ can be interpreted as the minimum second moment SDF in the hypothetical economy in which gross returns are $\mathbf{Z}$. The second components $\frac{1}{2} q_{0} \delta$ and $\frac{1}{2} q_{0}^{\star} \delta$ are orthogonal to $\mathbf{Z}$, where $\delta$ is normalized to have second moment equal to unity.

To illustrate that domestic and foreign investors disagree, in general, when $q_{0} \neq q_{0}^{\star}$, on their valuations of securities outside the linear span of $\mathbf{R}$ ( or $\mathbf{R}^{\star}$ ), consider, for example, a synthetic security that pays $\delta \sqrt{S_{t+1} / S_{t}}$ units of domestic currency, at time $t+1$. This would be privately valued, in domestic currency, at time $t$, at $\mathbb{E}_{t}\left[m \delta \sqrt{S_{t+1} / S_{t}}\right]=\mathbb{E}_{t}[y \delta]=\frac{1}{2} q_{0}$ by domestic investors and at $S_{t} \mathbb{E}_{t}\left[m^{\star} \delta\left(\sqrt{S_{t+1} / S_{t}}\right) / S_{t+1}\right]=S_{t} \mathbb{E}_{t}\left[y^{\star} \delta / S_{t}\right]=\frac{1}{2} q_{0}^{\star}$ by foreign investors.

If $q_{0}$ were to equal $q_{0}^{\star}$, these valuations would be the same, but $q_{0}=q_{0}^{\star}$ also implies that (i) $y=y^{*}$, and (ii) $m\left(\frac{S_{t+1}}{S_{t}}\right)-m^{\star}=0$.

The discrepancy between valuations of the synthetic security is greater when $\left|q_{0}-q_{0}^{\star}\right|$ is larger, which implies that $\left|y-y^{\star}\right|$ is larger and $\left|m\left(\frac{S_{t+1}}{S_{t}}\right)-m^{\star}\right|$ is larger. This is a situation that financial 
intermediaries may, potentially, wish to exploit, and they can do this by creating a synthetic security that offers payoffs outside the linear span of $\mathbf{R}$ (or $\mathbf{R}^{\star}$ ).

Our approach, broadly speaking, is to ask: By how much can domestic investors and foreign investors diverge on the valuation of securities outside the linear span of $\mathbf{R}$ (or $\mathbf{R}^{\star}$ ) before financial intermediaries would be presented with a "good deal"? The larger $\left|q_{0}-q_{0}^{\star}\right|$ is, the greater is the potential profit for financial intermediaries.

Hence, we study the consequences of a class of "good deals" characterized by

$$
q_{0}=-q_{0}^{\star} \equiv q \neq 0
$$

Specifically, equation (14), in conjunction with (12), translates into two restrictions on $y$ and $y^{*}$ :

$$
\begin{aligned}
\mathbb{E}_{t}\left[y-y^{*}\right] & =\frac{1}{2}\left(q_{0}-q_{0}^{*}\right) \mathbb{E}_{t}[\delta]=q \mathbb{E}_{t}[\delta], \\
\mathbb{E}_{t}\left[\left(y-y^{*}\right)^{2}\right] & =\frac{1}{4}\left(q_{0}-q_{0}^{*}\right)^{2} \mathbb{E}_{t}\left[\delta^{2}\right]=q^{2} .
\end{aligned}
$$

We use equations (15) and (16) to rule out implausibly high reward-for-risk strategies in the international economy with incomplete markets.

Consider now a financial intermediary that considers the possibility of privately negotiating a contract between itself and a domestic investor. We assume that the possible opportunity to enter into this private contract does not materially alter $m, m^{\star}, \mathbf{R}$, or $\mathbf{R}^{\star}$.

If entered into, the private contract with the domestic investor would require the investor to buy a synthetic security with payoff $x\left[\mathbf{Z}, S_{t+1}\right]$ in units of domestic currency, at time $t+1$, from the financial intermediary, where

$$
x\left[\mathbf{Z}, S_{t+1}\right]=\sqrt{S_{t+1} / S_{t}} \boldsymbol{w}^{\prime} \mathbf{Z}+\delta \sqrt{S_{t+1}} .
$$

Here, $\boldsymbol{w}$ is an $N$-dimensional vector of portfolio weights in the traded assets assumed to be of the form $\boldsymbol{w}=-\frac{1}{2}\left(q \sqrt{S_{t}}\right) \boldsymbol{v}$, where $\boldsymbol{v}^{\prime} \mathbf{1}=1$. 
The domestic investor computes the value, at time $t$, in units of domestic currency, of this synthetic security as:

$$
\begin{aligned}
\mathbb{E}_{t}\left[m\left(\sqrt{S_{t+1} / S_{t}} \boldsymbol{w}^{\prime} \mathbf{Z}+\delta \sqrt{S_{t+1}}\right)\right] & =\mathbb{E}_{t}\left[m\left(\boldsymbol{w}^{\prime} \mathbf{R}\right)\right]+\mathbb{E}_{t}\left[\left(y_{\mathbf{z}}+\frac{1}{2} q \delta\right) \delta \sqrt{S_{t}}\right] \\
& =\boldsymbol{w}^{\prime} \mathbf{1}+\frac{1}{2} q \sqrt{S_{t}}=0 .
\end{aligned}
$$

Thus, the cash flow postulated in (17) can be synthesized at zero cost.

If the financial intermediary were to enter into this private contract, it would have a short exposure to the cash flow $x\left[\mathbf{Z}, S_{t+1}\right]$ at time $t+1$. Substituting $\boldsymbol{w}=-\frac{1}{2}\left(q \sqrt{S_{t}}\right) \boldsymbol{v}$ into equation (17):

$$
\begin{aligned}
X & \equiv-x\left[\mathbf{Z}, S_{t+1}\right]=\frac{1}{2}\left(q \sqrt{S_{t}}\right) \boldsymbol{v}^{\prime} \mathbf{R}-\delta \sqrt{S_{t+1}}, \quad \text { (in units of domestic currency) } \\
& \left.=\frac{1}{2} q \boldsymbol{v}^{\prime} \mathbf{Z}-\delta, \quad \text { (in currency units of the hypothetical economy, i.e., } \sqrt{S_{t+1}}\right)
\end{aligned}
$$

where in moving from equation (19) to (20), we have divided by $\sqrt{S_{t+1}}$ because, since there are, at time $t+1, S_{t+1}$ units of domestic currency per unit of foreign currency, there are $\sqrt{S_{t+1}}$ units of domestic currency per unit of currency of the hypothetical economy (using equation (9)).

The financial intermediary faces a trade-off between the risks and rewards inherent in the cash flows $X$. We evaluate this trade-off in the currency units of the hypothetical economy to emphasize symmetry (without repeating the cash flow calculations in different currency units). Then,

$$
\begin{aligned}
\mathbb{E}_{t}[X] & =\frac{1}{2} q \mathbb{E}_{t}\left[\boldsymbol{v}^{\prime} \mathbf{Z}\right]-\mathbb{E}_{t}[\delta] \\
\mathbb{E}_{t}\left[\left(X-\mathbb{E}_{t}[X]\right)^{2}\right] & =\operatorname{Var}_{t}\left[\frac{1}{2} q \boldsymbol{v}^{\prime} \mathbf{Z}\right]+\mathbb{E}_{t}\left[\delta^{2}\right]-\left(\mathbb{E}_{t}[\delta]\right)^{2}-\underbrace{q \mathbb{E}_{t}\left[\boldsymbol{v}^{\prime} \mathbf{Z} \delta\right]}_{=0, \text { from eq. }}+q \mathbb{E}_{t}\left[\boldsymbol{v}^{\prime} \mathbf{Z}\right] \mathbb{E}_{t}[\delta] \\
& =\operatorname{Var}_{t}\left[\frac{1}{2} q \boldsymbol{v}^{\prime} \mathbf{Z}\right]+\mathbb{E}_{t}\left[\delta^{2}\right]-\left(\mathbb{E}_{t}[\delta]\right)^{2}+q \mathbb{E}_{t}\left[\boldsymbol{v}^{\prime} \mathbf{Z}\right] \mathbb{E}_{t}[\delta]
\end{aligned}
$$

In Appendix A, we show two results. First, $\left|\mathbb{E}_{t}\left[y-y^{\star}\right]\right|$ is always bounded above by an easily computable quantity that we argue will, in practice, be small. Second, in the special case that $m$, $m^{\star}$, and $S_{t+1} / S_{t}$ are lognormally distributed, $\mathbb{E}_{t}\left[y-y^{\star}\right]$ is identically equal to zero. Lognormality will only be an approximation to reality, but both results suggest that $\left|\mathbb{E}_{t}\left[y-y^{\star}\right]\right|$ will not be far from zero. From equation (15), this implies $\mathbb{E}_{t}[\delta] \approx 0$ given that $q \neq 0$. 
Using the approximation $\mathbb{E}_{t}[\delta] \approx 0$ in equation $(21)$, the expected payoff, denoted by $\mathrm{EP}$, to the financial intermediary can be approximated as:

$$
\begin{aligned}
\mathrm{EP} & \equiv \frac{1}{2} q \mathbb{E}_{t}\left[\boldsymbol{v}^{\prime} \mathbf{Z}\right] \\
& =\frac{1}{2} \mathbb{E}_{t}\left[\boldsymbol{v}^{\prime} \mathbf{Z}\right] \sqrt{\mathbb{E}_{t}\left[\left(y-y^{\star}\right)^{2}\right]}
\end{aligned}
$$

where we have substituted for $q$, using equation (16). The expression for EP in equation (24) is a measure of the potential reward to the financial intermediary. Analogously, by equation (23),

$$
\operatorname{Var}_{t}[X]-\operatorname{Var}_{t}\left[\frac{1}{2} q \boldsymbol{v}^{\prime} \mathbf{Z}\right] \approx \mathbb{E}_{t}\left[\delta^{2}\right]=1
$$

In words, the incremental variance of $X$ over and above that of the payoff $\frac{1}{2} q \boldsymbol{v}^{\prime} \mathbf{Z}$ is unity.

The quantity EP $/ \sqrt{\operatorname{Var}_{t}[X]-\operatorname{Var}_{t}\left[\frac{1}{2} q \boldsymbol{v}^{\prime} \mathbf{Z}\right]}=\mathrm{EP}$ is a measure of the reward-for-risk potentially available to the financial intermediary. It is equal to (or analogous to - definitions in the literature vary) what is variously termed (Sharpe (1981), Roll (1992), and Grinold and Kahn (2007)) the Information Ratio or the Appraisal Ratio (the latter being the term we will use), in that the reward is an excess return and the risk is measured as the square root of the incremental variance over and above that of a risky benchmark (in our setting, this risky benchmark is the portfolio with return $\frac{1}{2} q \boldsymbol{v}^{\prime} \mathbf{Z}$ ). This incremental variance is unity (by equation (25)).

If the reward-for-risk EP were high enough, a financial intermediary would have an incentive to privately negotiate contracts with investors that exploit the fact that domestic and foreign investors disagree on the valuations of securities outside the linear span of $\mathbf{R}$ ( or $\mathbf{R}^{\star}$ ).

We therefore place an upper bound on $\mathrm{EP} / \sqrt{\operatorname{Var}_{t}[X]-\operatorname{Var}_{t}\left[\frac{1}{2} q \boldsymbol{v}^{\prime} \mathbf{Z}\right]}$, which has the effect of ruling out a potential contract that is too good to be true (e.g., Cochrane and Saá-Requejo (2000)).

Specifically, for some $\widehat{\Theta}$, satisfying $0 \leq \widehat{\Theta}<+\infty$, the reward-for-risk is bounded:

$$
|\mathrm{EP}|=\left|\frac{1}{2} \mathbb{E}\left[\boldsymbol{v}^{\prime} \mathbf{Z}\right] \sqrt{\mathbb{E}_{t}\left[\left(y-y^{*}\right)^{2}\right]}\right| \leq \widehat{\Theta} .
$$


Alternatively, defining $\Theta$ by $\mathbb{E}_{t}\left[v^{\prime} \mathbf{Z}\right] \Theta \equiv 2 \widehat{\Theta}$, and substituting into equation (26) and henceforth, for brevity, dropping the subscript $t$ from the expectation operator, we exclude good deals in the international economy by placing an upper bound $\Theta$ as follows:

$$
\mathbb{E}\left[\left(y-y^{\star}\right)^{2}\right] \leq \Theta^{2}
$$

for some economically- and empirically motivated choice of $\Theta$ (for $0 \leq \Theta<+\infty$ ). Equation (27) places a restriction on $y$ and $y^{\star}$ and, thus, on the set of admissible $m$ and $m^{\star}$.

Equation (27) is central to our analysis, and can be distinguished from the setting where $m\left(\frac{S_{t+1}}{S_{t}}\right)-m^{\star}=0$, which entails $y-y^{\star}=0$, which in turn, imposes $\mathbb{E}\left[\left(y-y^{\star}\right)^{2}\right]=0$. One may view $\Theta$ as quantifying deviations from market completeness.

We will refer to $\Theta$ as the market incompleteness parameter and outline its identification within the context of our empirical work.

\subsection{Operationalizing the framework when $m\left(\frac{S_{t+1}}{S_{t}}\right)-m^{\star}$ need not equal zero}

In complete markets, $m\left(\frac{S_{t+1}}{S_{t}}\right)-m^{\star}=0$ tightly links exchange rate growth and $m$ and $m^{\star}$. Moreover, Brandt, Cochrane, and Santa-Clara (2006) show that the minimum variance $m$ and $m^{\star}$ recovered from asset return data, also satisfy $m\left(\frac{S_{t+1}}{S_{t}}\right)-m^{\star}=0$ in an incomplete market. Their analysis further reveals that with $m\left(\frac{S_{t+1}}{S_{t}}\right)-m^{\star}=0$ imposed, the risk sharing index, based on asset return data (defined in their equation (2)), is computed to be high, indicating a high degree of international risk sharing, whereas risk sharing based on consumption growth data is low.

In contrast, we study a theory that allows for a multitude of SDFs in incomplete markets and seeks to synthesize $m$ and $m^{\star}$ that are restricted by a feasible set (outlined shortly) that may or may not be consistent with $m\left(\frac{S_{t+1}}{S_{t}}\right)-m^{\star}=0$, and simultaneously offers flexibility in producing patterns of limited international risk sharing. Thus, we ask, if we restrict our attention to $\left(m, m^{\star}\right)$ pairs, which are economically plausible and consistent with equations (5), (6), and (7), how low can the risk sharing index, based on asset return data, be?

Or, how low can $\operatorname{Cov}\left[m, m^{\star}\right]$ be? Is it low enough to be reconcilable with the realities of international risk sharing based on consumption growth data? 
To proceed with our goal of deducing the implications of consumption and asset return data for SDFs in incomplete markets, we work in discrete time, and consider specifying a risk sharing index, analogous to that of Brandt, Cochrane, and Santa-Clara (2006, equation (2)), of the form

$$
\operatorname{RSI} \equiv \frac{2 \operatorname{Cov}\left[m, m^{\star}\right]}{\operatorname{Var}[m]+\operatorname{Var}\left[m^{\star}\right]}=\frac{2 \mathbb{E}\left[m m^{\star}\right]-2 /\left(R_{f} R_{f}^{\star}\right)}{\operatorname{Var}[m]+\operatorname{Var}\left[m^{\star}\right]}
$$

In the setting of incomplete markets, there is an infinite number of $m$ and $m^{\star}$ and, thus, an infinite number of possible values of such a proposed risk sharing index, which leads us to possibly take infimums, over $m$ and $m^{\star}$, of a risk sharing index in equation (28).

Thus, in essence, we are asking what is a plausible, but economically justified, lower bound on the covariances (correlations) between SDFs based on available data?

To operationalize our incomplete markets framework, we compute the RSI in (28). This calculation is important to our approach, as it allows us to estimate the market incompleteness parameter $\Theta$ by aligning estimates of international risk sharing, based on asset returns, with those from consumption growth, details of which are provided in Section 3.1.

Recalling from (10) that $y \equiv m \sqrt{S_{t+1} / S_{t}}$ and $y^{\star} \equiv m^{\star} / \sqrt{S_{t+1} / S_{t}}$, we consider the following problem (which, since $y y^{\star}=m m^{\star}$, is equivalent to the objective $\inf _{m, m^{\star}} \mathbb{E}\left[2 m m^{\star}\right]$ ):

Problem 1 Choose $y$ and $y^{\star}$ to

$$
\inf _{y, y^{\star}} \mathbb{E}\left[2 y y^{\star}\right]
$$

subject to

$$
\begin{array}{ll}
\mathbb{E}\left[\left(y-y^{\star}\right)^{2}\right] \leq \Theta^{2}, & \text { (relaxes the restriction that } \left.m\left(\frac{S_{t+1}}{S_{t}}\right)-m^{\star}=0\right) \\
\mathbb{E}[y \boldsymbol{Z}]=\mathbb{E}\left[y^{\star} \boldsymbol{Z}\right]=\mathbf{1}, & \text { (correct pricing) } \\
y \geq 0 \text { and } y^{\star} \geq 0 . & \text { (nonnegativity constraints) }
\end{array}
$$

In Problem 1, the inequality constraint (30) arises as a consequence of incorporating the incomplete markets assumption in the international economy, whereby $m\left(\frac{S_{t+1}}{S_{t}}\right)-m^{\star}$ need not equal zero. 
Specifically, $\Theta^{2}$ is the upper bound on $\mathbb{E}\left[\left(y-y^{\star}\right)^{2}\right]$. The equality constraint $\mathbb{E}[y \mathbf{Z}]=\mathbb{E}\left[y^{\star} \mathbf{Z}\right]=\mathbf{1}$ in equation (31) is equivalent to $\mathbb{E}[m \mathbf{R}]=\mathbf{1}$ and $\mathbb{E}\left[m^{\star} \mathbf{R}^{\star}\right]=\mathbf{1}$ and enforces that $m$ and $m^{\star}$ must price the returns $\mathbf{R}$ and $\mathbf{R}^{\star}$.

We are interested in analyzing what incomplete markets have to say about SDF volatilities and international finance puzzles. At the same time, we are interested in inferring marginal utility growth rates that are consistent with the data. The marginal utilities are nonnegative, so, following Hansen and Jagannathan (1991), we focus on nonnegative SDFs in the admissible set. The constraints $y \geq 0$ and $y^{\star} \geq 0$ in equation (32) are equivalent to $m \geq 0$ and $m^{\star} \geq 0$.

In the objective function (29), we essentially compute a lower bound on the value of the numerator of equation (28) consistent with repricing the returns $\mathbf{R}$ and $\mathbf{R}^{\star}$, consistent with the absence of arbitrage, and consistent with the upper bound $\mathbb{E}\left[\left(y-y^{\star}\right)^{2}\right] \leq \Theta^{2}$.

Still, the optimization problem could become ill-posed if one could find $m$ and $m^{\star}$, where the objective is unbounded. Such an outcome is disallowed with our constraints and via $\left|\mathbb{E}\left[y y^{*}\right]\right|<+\infty$. The solution depends critically on $\mathbb{E}\left[\left(y-y^{\star}\right)^{2}\right] \leq \Theta^{2}$.

Observe further that if, instead, we were to minimize the ratio $2 \operatorname{Cov}\left[m, m^{\star}\right] /\left(\operatorname{Var}[m]+\operatorname{Var}\left[m^{\star}\right]\right)$ subject to the constraints in equations (30) through (32), we would obtain a value of the risk sharing index that is (weakly) lower than that obtained from Problem 1.

\subsection{Solving the problem}

Based on the problem in equations (29)-(32), we look for solutions for $y$ and $y^{\star}$ of the form

$$
y=y_{\mathbf{z}}+\frac{1}{2} d \Theta \delta, \quad \text { and } \quad y^{\star}=y_{\mathbf{z}}+\frac{1}{2} d^{\star} \Theta \delta
$$

where $y_{\mathbf{z}} \geq 0, \delta, d$, and $d^{*}$ are yet to be determined (the conjectured solution inherits the form in equation (12), but with $q_{0}=d \Theta$ and $\left.q_{0}^{*}=d^{*} \Theta\right)$.

For now, $d$ and $d^{\star}$ are constant scalars satisfying $\left|d-d^{\star}\right| \leq 2$. Further the random variables $y_{\mathbf{z}}$ and $\delta$ satisfy

$$
\mathbb{E}\left[y_{\mathbf{z}} \delta\right]=0, \quad \mathbb{E}[\delta \mathbf{Z}]=0 \text { for each element of } \mathbf{Z}, \quad \text { and } \quad \mathbb{E}\left[\delta^{2}\right]=1 .
$$


Because $y-y^{\star}=\frac{1}{2}\left(d-d^{\star}\right) \Theta \delta$, the constraint $\mathbb{E}\left[\left(y-y^{\star}\right)^{2}\right] \leq \Theta^{2}$ is automatically satisfied. The constraint $\mathbb{E}[y \mathbf{Z}]=\mathbb{E}\left[y^{\star} \mathbf{Z}\right]=\mathbf{1}$ in equation (31) is satisfied, provided $\mathbb{E}\left[y_{\mathbf{z}} \mathbf{Z}\right]=\mathbf{1}$.

With the conjectured forms of $y$ and $y^{\star}$ in equation (33), $\mathbb{E}\left[m m^{\star}\right]=\mathbb{E}\left[y y^{\star}\right]=\mathbb{E}\left[y_{\mathbf{z}}^{2}\right]+\frac{1}{4} d d^{\star} \Theta^{2}$. Hence, the infimum $\inf _{y, y^{\star}} \mathbb{E}\left[y y^{\star}\right]$ in Problem 1 separates into two distinct problems:

$$
\inf _{y_{\mathbf{z}}}\left\{\mathbb{E}\left[y_{\mathbf{z}}^{2}\right]\right\}+\inf _{d, d^{\star}}\left\{\frac{1}{4} d d^{\star} \Theta^{2}\right\}
$$

subject to $\mathbb{E}\left[y_{\mathbf{z}} \mathbf{Z}\right]=\mathbf{1}, y_{\mathbf{z}} \geq 0, y \geq 0$, and $y^{\star} \geq 0$.

Exploiting this feature of the solution, we sequentially solve for $y_{\mathbf{z}}$, then for $\delta$ and, finally, for $d$ and $d^{\star}$. We first determine $y_{\mathbf{z}}$ by solving

$$
\inf _{y_{\mathbf{z}}} \mathbb{E}\left[y_{\mathbf{z}}^{2}\right] \quad \text { such that } \quad \mathbb{E}\left[y_{\mathbf{z}} \mathbf{Z}\right]=\mathbf{1}, \quad y_{\mathbf{z}} \geq 0
$$

Here, $y_{\mathbf{z}}$ can be interpreted as the minimum second moment SDF with nonnegativity in the hypothetical economy in which gross returns are $\mathbf{Z}$.

Introduce an $N$-dimensional vector of Lagrange multipliers $\boldsymbol{\lambda}$. Then the solution to the problem in equation (36) is the solution to

$$
\max _{\boldsymbol{\lambda}}\left\{\inf _{y_{\mathbf{z}} \geq 0}\left\{\mathbb{E}\left[y_{\mathbf{z}}^{2}\right]-2 \boldsymbol{\lambda}^{\prime}\left(\mathbb{E}\left[y_{\mathbf{z}} \mathbf{Z}\right]-\mathbf{1}\right)\right\}\right\}
$$

The first-order condition implies $0=2 y_{\mathbf{z}}-2 \lambda^{\prime} \mathbf{Z}$. Both the first-order condition and the constraint $y_{\mathbf{z}} \geq 0$ will be satisfied if

$$
y_{\mathbf{z}}=\max \left(\boldsymbol{\lambda}^{\prime} \mathbf{Z}, 0\right)
$$

Substituting $y_{\mathbf{z}}$ from equation (38) into equation (37) $\boldsymbol{\lambda}$ solves

$$
\max _{\boldsymbol{\lambda}}\left\{2 \boldsymbol{\lambda}^{\prime} \mathbf{1}-\mathbb{E}\left[\left(\max \left(\boldsymbol{\lambda}^{\prime} \mathbf{Z}, 0\right)\right)^{2}\right]\right\}, \text { or, equivalently, } \quad-\min _{\boldsymbol{\lambda}}\left\{\mathbb{E}\left[\left(\max \left(\boldsymbol{\lambda}^{\prime} \mathbf{Z}, 0\right)\right)^{2}\right]-2 \boldsymbol{\lambda}^{\prime} \mathbf{1}\right\}
$$

Next, to solve for $\delta$, we note that $\delta$ is proportional to $e_{\mathbf{z}}$, where $e_{\mathbf{z}}$ is the residual from the projection of one onto the space of returns $\mathbf{Z}$. Hence, $e_{\mathbf{z}}$ can be computed from the Ordinary Least Squares regression formula (e.g., Cochrane (2005, page 95)). 
Then $\delta$ is obtained by multiplicatively scaling $e_{\mathrm{z}}$ in such a way that $\mathbb{E}\left[\delta^{2}\right]=1$. More formally,

$$
e_{\mathbf{z}}=1-\mathbb{E}[\mathbf{Z}]^{\prime}\left(\mathbb{E}\left[\mathbf{Z} \mathbf{Z}^{\prime}\right]\right)^{-1} \mathbf{Z}, \quad \text { and then } \quad \delta=e_{\mathbf{z}} / \sqrt{\mathbb{E}\left[e_{\mathbf{z}}^{2}\right]}
$$

In the degenerate case that $e_{\mathbf{z}}=0$ in every state, we set $\delta=0$. Our characterization of $\delta$ presented in equation (40) is aimed at partitioning the SDF into distinct portions that are spanned, and that are unspanned, by the available set of asset returns.

Finally, we solve for $d$ and $d^{\star}$. The second part of equation (35) minimizes $\frac{1}{4} d d^{\star} \Theta^{2}$, and the minimum requires that $d$ and $d^{\star}$ be of opposite signs. Hence, without loss of generality, we assume

$d \geq 0, d^{\star} \leq 0$. The solution of Problem 1 must also accommodate $y=y_{\mathbf{z}}+\frac{1}{2} d \Theta \delta \geq 0$ and $y^{\star}=y_{\mathbf{z}}+\frac{1}{2} d^{\star} \Theta \delta \geq 0$.

Let $d_{p}$ (respectively, $d_{n}$ ) be the smallest positive value (respectively, largest negative, i.e., least negative value) of $-y_{\mathbf{z}} /\left(\frac{1}{2} \Theta \delta\right)$ across the $\mathbb{I}$ possible states of the world. Then $y \geq 0$, and $y^{\star} \geq 0$ requires that $d \leq d_{p}$ and $d_{n} \leq d^{\star}$.

Taken together with the constraint $d-d^{\star} \leq 2$, the solution for $d$ and $d^{\star}$ can be summarized as

$$
\left\{\begin{array}{l}
\text { If } d_{p} \geq 1 \text { and } d_{n} \leq-1, \text { then } d=1 \text { and } d^{\star}=-1 \\
\text { else } d=\min \left(d_{p},-d_{n}\right) \text { and } d^{\star}=-d
\end{array}\right.
$$

These values for $d$ and $d^{\star}$, together with equations (39) and (40), provide the values of $y$ and $y^{\star}$ and, thus, of $m=y / \sqrt{S_{t+1} / S_{t}}$ and $m^{\star}=y^{*} \sqrt{S_{t+1} / S_{t}}$, which solve Problem 1 .

\subsection{The big picture and characterizing the unspanned components of $m$ and $m^{*}$}

The advantage of the solution for $y$ and $y^{*}$ in equation (33) is that it facilitates the characterization of the spanned and unspanned components of the SDFs:

$$
m_{t+1}=\underbrace{m_{\mathbf{z}, t+1}}_{\text {spanned }}+\underbrace{u_{t+1}}_{\text {unspanned }}, \quad \text { and } \quad m_{t+1}^{*}=\underbrace{m_{\mathbf{z}, t+1}^{*}}_{\text {spanned }}+\underbrace{u_{t+1}^{*}}_{\text {unspanned }} \text {. }
$$


We recognize that the extraction of the spanned and unspanned components is consistent with the objective $\inf _{m, m^{\star}} \mathbb{E}\left[2 \mathrm{~m} \mathrm{~m}^{\star}\right]$, consistent with ruling out reward-for-risk perceived to be unacceptable in international economies, and consistent with the nonnegativity of SDFs. More explicitly,

$$
\begin{aligned}
& m_{\mathbf{z}} \equiv \frac{y_{\mathbf{z}}}{\sqrt{S_{t+1} / S_{t}}}=\frac{1}{\left(S_{t+1} / S_{t}\right)} \max \left(\boldsymbol{\lambda}^{\prime} \mathbf{R}, 0\right), \quad \text { and } \quad u \equiv \frac{1}{2}(d \Theta \delta) \frac{1}{\sqrt{S_{t+1} / S_{t}}}, \\
& m_{\mathbf{z}}^{*} \equiv y_{\mathbf{z}} \sqrt{S_{t+1} / S_{t}}=\left(S_{t+1} / S_{t}\right) \max \left(\boldsymbol{\lambda}^{\prime} \mathbf{R}^{*}, 0\right), \quad \text { and } \quad u^{*} \equiv \frac{1}{2}\left(d^{\star} \Theta \delta\right) \sqrt{S_{t+1} / S_{t}},
\end{aligned}
$$

where $\boldsymbol{\lambda}$ solves (39) and $\delta$ is determined via (40). We will establish that $\mathbb{E}_{t}[u]=\mathbb{E}_{t}\left[u^{*}\right]=0$.

We shall refer to the characterization in equation (42) as the additive form of the SDFs.

Our depiction of the spanned and unspanned components of SDFs in (43) and (44) - which are consistent with the lower bound on SDF covariances - furnishes new insights:

- As derived, the properties of $u$ and $u^{*}$ hinge critically on $\Theta$. For instance, a higher value of $\Theta$ has the effect of increasing the volatility of $u$ and $u^{*}$ and, therefore, of $m$ and $m^{*}$. In contrast, the volatility of both $m_{\mathbf{z}}$ and $m_{\mathbf{z}}^{*}$ is invariant to $\Theta$.

- Next, $\operatorname{Cov}\left[u, u^{*}\right]=\frac{1}{4} d d^{\star} \Theta^{2} \leq 0$ (we have used equation (34) along with $\mathbb{E}[u]=\mathbb{E}\left[u^{*}\right]=0$ ), which is negative given our objective $\inf _{d, d^{\star}}\left\{\frac{1}{4} d d^{\star} \Theta^{2}\right\}$ and the result that $d$ and $d^{*}$ are of the opposite sign. Moreover, the magnitude of $\operatorname{Cov}\left[u, u^{*}\right]$ is dictated by the data on international risk sharing and asset returns. Hence, $d d^{\star}<0$ is not mechanically imposed.

These derived attributes of our solution provide the intuition for the ensuing quantitative assessments regarding both the risk sharing index and the correlation between $m$ and $m^{*}$ :

$$
\mathrm{RSI}=\frac{2 \operatorname{Cov}\left[m, m^{\star}\right]}{\operatorname{Var}[m]+\operatorname{Var}\left[m^{\star}\right]} \approx \frac{2 \operatorname{Cov}\left[m_{\mathbf{z}}, m_{\mathbf{z}}^{*}\right]+2 \operatorname{Cov}\left[u, u^{*}\right]}{\operatorname{Var}\left[m_{\mathbf{z}}\right]+\operatorname{Var}\left[m_{\mathbf{z}}^{*}\right]+\operatorname{Var}[u]+\operatorname{Var}\left[u^{*}\right]} .
$$

Thus, a higher $\Theta$ attenuates RSI by making $\operatorname{Cov}\left[u, u^{*}\right]$ more negative. We note additionally that imposing $\Theta=0$ translates into $u=u^{*}=0$ and is isomorphic to a high RSI.

One may be able to garner a better conceptual understanding of our solution mechanism by drawing on the work of others. In Kim and Schiller (2015), for example, the economies are inhabited by both stockholders and non-stockholders. Since the stockholders have access to capital markets, they are able to achieve high risk sharing, with two consequences. First, the consumption growths 
of stockholders in the domestic and foreign countries are intrinsically linked. Second, the marginal utilities are highly correlated. At the same time, market incompleteness is introduced because the non-stockholders can only trade in a bond. Such a friction can hinder the ability of non-stockholders to share adverse economic shocks. The salient outcome is that aggregate consumption (stockholder plus non-stockholder) growth can be moderately correlated, whereas the stockholders consumption growth and SDFs can be sizably correlated. In our paper, we have offered a different tractable framework in which market incompleteness permeates throughout the economy.

The decomposition articulated in equations (43) and (44) allows us to study the implications of incomplete markets for the behavior of exchange rate growth, as presented in equation (4):

$$
\begin{aligned}
0 & =\mathbb{E}_{t}\left[m\left(S_{t+1} / S_{t}\right)-m^{\star}\right], \\
& =\mathbb{E}_{t}[\underbrace{\max \left(\boldsymbol{\lambda}^{\prime} \mathbf{R}, 0\right)-\left(S_{t+1} / S_{t}\right) \max \left(\boldsymbol{\lambda}^{\prime} \mathbf{R}^{*}, 0\right)}_{=0, \text { since } \mathbf{R}_{t+1}=\left(S_{t+1} / S_{t}\right) \mathbf{R}_{t+1}^{\star}}+\frac{\sqrt{S_{t+1} / S_{t}}}{2} d \Theta \delta-\frac{\sqrt{S_{t+1} / S_{t}}}{2} d^{*} \Theta \delta], \\
& \left.=\frac{1}{2}\left(d-d^{*}\right) \Theta \mathbb{E}_{t}\left[\delta \sqrt{S_{t+1} / S_{t}}\right] . \quad \quad \text { (note that } d-d^{*} \neq 0 \text { and } \Theta \neq 0\right)
\end{aligned}
$$

Thus, we obtain $\mathbb{E}_{t}\left[u^{*}\right]=0$, and likewise $0=\mathbb{E}_{t}\left[m-m^{\star} /\left(\frac{S_{t+1}}{S_{t}}\right)\right]$ implies that $\mathbb{E}_{t}[u]=0$.

Viewed in conjunction with the solution of $\delta$ from equation (40) and setting $\boldsymbol{\alpha} \equiv\left(\mathbb{E}\left[\mathbf{Z Z} \mathbf{Z}^{\prime}\right]\right)^{-1} \mathbb{E}[\mathbf{Z}]$, the conditions $\mathbb{E}_{t}[u]=0$ and $\mathbb{E}_{t}\left[u^{*}\right]=0$ manifest the following restrictions on exchange rate growth:

$$
\mathbb{E}_{t}\left[\left(1-\boldsymbol{\alpha}^{\prime} \mathbf{Z}_{t+1}\right) / \sqrt{S_{t+1} / S_{t}}\right]=0, \quad \text { and also } \quad \mathbb{E}_{t}\left[\left(1-\boldsymbol{\alpha}^{\prime} \mathbf{Z}_{t+1}\right) \sqrt{S_{t+1} / S_{t}}\right]=0
$$

The implication is that $\left(1-\boldsymbol{\alpha}^{\prime} \mathbf{Z}_{t+1}\right) / \sqrt{S_{t+1} / S_{t}}$ and $\left(1-\boldsymbol{\alpha}^{\prime} \mathbf{Z}_{t+1}\right) \sqrt{S_{t+1} / S_{t}}$ are orthogonal to time $t$ information variables, with zero regression coefficients (e.g., Hansen and Hodrick (1980)).

\subsection{Exploring complementarities and differences from other approaches}

Is our solution in equation (42) subsumed within the multiplicative wedge class?

To offer our rationale, consider Lustig and Verdelhan (2016, pages 8 and 9), where an econometrician commits to a model of "base case" SDFs. The foreign base case SDF is then "perturbed" by a multiplicative wedge $e^{\eta_{t+1}}$, satisfying $m_{t+1}\left(\frac{S_{t+1}}{S_{t}}\right)=m_{t+1}^{*} e^{\eta_{t+1}}$. In analogy, our base case SDFs are $m_{\mathbf{z}, t+1}=\frac{y_{\mathbf{z}}}{\sqrt{S_{t+1} / S_{t}}}$ (domestic) and $m_{\mathbf{z}, t+1}^{*}=y_{\mathbf{z}} \sqrt{S_{t+1} / S_{t}}$ (foreign), defined in (43) and (44), 
since complete markets would correspond to the case when the only (i.e., unique) SDFs satisfying (5)-(7) were of this form. Recall that $y_{\mathbf{z}}$ is interpretable as a minimum second moment SDF.

Our analysis leads to consideration of domestic and foreign SDFs of the form $m=y / \sqrt{S_{t+1} / S_{t}}$ and $m^{\star}=y^{\star} \sqrt{S_{t+1} / S_{t}}$, where $y$ and $y^{\star}$ are of the form $y=y_{\mathbf{z}}+\frac{1}{2} d \Theta \delta$ and $y^{\star}=y_{\mathbf{z}}+\frac{1}{2} d^{\star} \Theta \delta$ (see equation (33)). Given the symmetric construction, it is clear that one would need to "perturb" not only the base case foreign SDF, but also the base case domestic SDF.

However, even if one were to consider "perturbed" SDFs of the form $m_{t+1} e^{-h \eta_{t+1}}$ and $m_{t+1}^{*} e^{(1-h) \eta_{t+1}}$ (consistent with $m_{t+1}\left(\frac{S_{t+1}}{S_{t}}\right)=m_{t+1}^{*} e^{\eta_{t+1}}$ ), for some constant scalar $h$, one still cannot obtain the additive SDFs proposed in our paper. To obtain such SDFs, one would need $e^{-h \eta_{t+1}} m_{\mathbf{z}, t+1}=$ $\left(y_{\mathbf{z}}+\frac{1}{2} d \Theta \delta\right) / \sqrt{S_{t+1} / S_{t}}$ and, simultaneously, $e^{(1-h) \eta_{t+1}} m_{\mathbf{z}, t+1}^{*}=\left(y_{\mathbf{z}}+\frac{1}{2} d^{\star} \Theta \delta\right) \sqrt{S_{t+1} / S_{t}}$. Or, equivalently, $e^{(1-h) \eta_{t+1}}=\left(\left(y_{\mathbf{z}}+\frac{1}{2} d \Theta \delta\right) / y_{\mathbf{z}}\right)^{-(1-h) / h}=\left(y_{\mathbf{z}}+\frac{1}{2} d^{\star} \Theta \delta\right) / y_{\mathbf{z}}$. But the right-hand side equality is not mathematically feasible except when $d \Theta=d^{\star} \Theta=0$ (which leads back to the base case SDFs).

In particular, with base case SDFs of the form $m_{\mathbf{z}, t+1} \equiv \frac{y_{\mathbf{z}}}{\sqrt{S_{t+1} / S_{t}}}$ and $m_{\mathbf{z}, t+1}^{*} \equiv y_{\mathbf{z}} \sqrt{S_{t+1} / S_{t}}$, the relation $m_{t+1}\left(\frac{S_{t+1}}{S_{t}}\right)=m_{t+1}^{*} e^{\eta_{t+1}}$ implies $\frac{y_{\mathbf{z}}}{\sqrt{S_{t+1} / S_{t}}}\left(\frac{S_{t+1}}{S_{t}}\right)=y_{\mathbf{z}} \sqrt{S_{t+1} / S_{t}} e^{\eta_{t+1}}$ or $1=e^{\eta_{t+1}}$ or $\eta_{t+1}=0$. Thus, not all SDFs - and certainly not the SDFs that we synthesize - can be tailored to be in line with the multiplicative wedge paradigm.

Expanding, the approach of Lustig and Verdelhan does not allow consideration of the class of SDFs that we show are compatible with low correlations between the SDFs as well as limited risk sharing. As noted in Bakshi and Crosby (2016), the multiplicative wedge with incomplete spanning approach does not affect the correlation between the SDFs. These are essential matters from the standpoint of our empirical conclusions.

Overall, the departure from the extant literature is our treatment that enables the closed-form tractability of the spanned and unspanned components of the SDFs. Moreover, our analysis wards off a possible misconception that multiplicative wedges subsume our additive form of the SDFs. The additive form of the SDFs is implicit in the constructions of Hansen and Jagannathan (1991).

Finally, we solve a particularly parameterized economy with five states in the Internet Appendix (Section I and Table Internet-I), which helps to synthesize, in a simplified setting, the various elements of our approach, to study SDF correlations and volatilities under incomplete markets. 


\section{What does our approach tell us about international economies?}

Our empirical investigation employs data on consumption, bonds, and broad-based equity indexes for 10 countries - namely, Australia (AUD), New Zealand (NZD), United Kingdom (STG), France (FRA), Canada (CAD), United States (USD), Netherlands (NLG), Germany (GER), Japan (JPY), and Switzerland (SWI). The sample period is January 1975 to June 2014 (474 observations).

When computing, for example, the volatilities and correlations of SDFs that are supported in our incomplete markets framework, for Australia and Japan, the gross return vector $\mathbf{R}_{t+1}$ includes real returns on six assets, namely, on the Australian risk-free bond, on the Australian equity index, on the Japanese risk-free bond, on the Japanese equity index, on the U.S. 30-year Treasury bond, and on the MSCI world equity index, all denominated in Australian dollars, while $\mathbf{R}_{t+1}^{\star}$ includes returns on the same six assets, but now all the real returns are denominated in Japanese yen.

Moreover, the choice of assets in $\mathbf{R}_{t+1}$ (or $\mathbf{R}_{t+1}^{*}$ ) embed multi-currency and multi-country exposures. Later, we assess the effect of enhancing (or reducing) the dimensionality of $\mathbf{R}_{t+1}$ (or $\left.\mathbf{R}_{t+1}^{*}\right)$ on the properties of the SDFs pairs $\left(m_{t+1}, m_{t+1}^{*}\right)$.

The country-specific data on the LIBOR interest-rate, equity index, exchange rates, consumption growth, and inflation are described in Section II of the Internet Appendix.

\subsection{Identification and admissible values of $\Theta$}

At the front and center of our theory is the feature that a higher market incompleteness parameter $\Theta$ is associated with a greater volatility of the unspanned components of the SDFs. In this regard, the constraint $\mathbb{E}\left[\left(y-y^{\star}\right)^{2}\right] \leq \Theta^{2}$, in equation (27), is pivotal to our characterizations in incomplete markets, but leaves open the question of how to identify and estimate $\Theta$.

\subsubsection{Motivating an algorithm for identifying $\Theta$}

Our identification strategy for $\Theta$ involves the consideration of two crucial theoretical objects and one salient and pervasive attribute of international macroeconomic data. 
First, to establish the reasonableness of $\Theta$, we exploit the closed-form tractability of $\sqrt{\operatorname{Var}\left[m_{t+1}\right]}$, which we compute via equations (42) and (43) as

$$
\operatorname{Var}\left[m_{t+1}\right] \approx \underbrace{\operatorname{Var}\left[m_{\mathbf{z}, t+1}\right]}_{\text {independent of } \Theta}+\underbrace{\mathbb{E}\left[u_{t+1}^{2}\right]}_{\text {varies with } \Theta} \quad\left(\text { since } \mathbb{E}\left[u_{t+1}\right]=0, \operatorname{Cov}\left[m_{\mathbf{z}, t+1}, u_{t+1}\right] \approx 0\right) .
$$

Second, departures from complete markets can translate into less correlated SDFs, as in

$$
\underbrace{\rho_{m, m^{*}}}_{\text {correlation }} \equiv \underbrace{\frac{\operatorname{Cov}\left[m_{\mathbf{z}, t+1}, m_{\mathbf{z}, t+1}^{*}\right]+\operatorname{Cov}\left[u_{t+1}, u_{t+1}^{*}\right]}{\sqrt{\operatorname{Var}\left[m_{t+1}\right] \operatorname{Var}\left[m_{t+1}^{*}\right]}}}_{\text {varies with } \Theta}
$$

The insight to garner is that there is a trade-off between the volatility of the SDFs and the correlation $\rho_{m, m^{*}}$, namely, a higher $\Theta$ increases the volatility of the unspanned component of the SDFs but lowers the covariance and, typically, lowers the correlation between the SDFs.

Our theory argues that a financial intermediary would have an incentive to privately negotiate contracts with investors that exploit the fact that domestic and foreign investors disagree on the valuations of securities outside the linear span of $\mathbf{R}_{t+1}$ ( or $\mathbf{R}_{t+1}^{\star}$ ), if the potential reward-for-risk were to be high. The domestic and foreign SDFs become less correlated with larger divergence between the valuations of securities outside the linear span of $\mathbf{R}_{t+1}$ (or $\mathbf{R}_{t+1}^{\star}$ ).

In contrast, a theory with $\Theta=0$ implies $m_{t+1}\left(\frac{S_{t+1}}{S_{t}}\right)=m_{t+1}^{\star}$, and domestic and foreign investors would place identical valuation on all Arrow-Debreu securities irrespective of whether they trade. It also runs counter to the intuition that, if consumption growths are imperfectly correlated, then unspanned states that are relatively unpleasant (favorable) for domestic (foreign) investors would result in domestic investors placing greater marginal utility on them than foreign investors.

Third, germane to our identification strategy is the consensus that correlations between consumption growth in industrialized countries are low. Exploiting this link, together with an analysis of SDF volatilities and correlations, allows us to identify admissible values of $\Theta$. Our aim is to ask whether the identified $\Theta$ is consistent with the dimensions of consumption and asset return data. A body of literature has sought to reconcile these two aspects of the data, as laid out, among others, in Lewis (1996), Colacito and Croce (2011, 2013), Gavazzoni, Sambalaibat, and Telmer (2013), Colacito, Croce, Gavazzoni, and Ready (2015), Stathopoulos (2015), and Zhang (2015). 
Fleshing out our identification strategy leads us to define the consumption-based risk sharing index as

$$
\operatorname{RSI}^{C} \equiv \frac{2 \operatorname{Cov}\left[\log \left(c_{t+1} / c_{t}\right), \log \left(c_{t+1}^{\star} / c_{t}^{\star}\right)\right]}{\operatorname{Var}\left[\log \left(c_{t+1} / c_{t}\right)\right]+\operatorname{Var}\left[\log \left(c_{t+1}^{\star} / c_{t}^{\star}\right)\right]}
$$

To understand (51), assume a marginal rate of substitution consistent with isoelastic utility and the same risk aversion $\gamma$ in each country (as in Backus and Smith (1993) and Brandt, Cochrane, and Santa-Clara (2006, equation (3))). Consider the approximation $\left(c_{t+1} / c_{t}\right)^{-\gamma}=\exp \left(-\gamma \log \left(c_{t+1} / c_{t}\right)\right) \approx$ $1-\gamma \log \left(c_{t+1} / c_{t}\right)$, so $\operatorname{Var}\left[\left(c_{t+1} / c_{t}\right)^{-\gamma}\right] \approx \gamma^{2} \operatorname{Var}\left[\log \left(c_{t+1} / c_{t}\right)\right], \operatorname{Var}\left[\left(c_{t+1}^{*} / c_{t}^{*}\right)^{-\gamma}\right] \approx \gamma^{2} \operatorname{Var}\left[\log \left(c_{t+1}^{*} / c_{t}^{*}\right)\right]$, and $\operatorname{Cov}\left[\left(c_{t+1} / c_{t}\right)^{-\gamma},\left(c_{t+1}^{*} / c_{t}^{*}\right)^{-\gamma}\right] \approx \gamma^{2} \operatorname{Cov}\left[\log \left(c_{t+1} / c_{t}\right), \log \left(c_{t+1}^{\star} / c_{t}^{\star}\right)\right]$. Canceling $\gamma^{2}$, we get $\operatorname{RSI}^{C}$.

One implication of incomplete markets is that $\mathrm{RSI}^{C}$ will not be high, as noted and elaborated in Backus and Smith (1993, page 298), Brandt, Cochrane, and Santa-Clara (2006), Lewis and Liu (2015), and Zhang (2015). Across our sample of 45 country pairs, the average $\mathrm{RSI}^{C}$ is $26 \%$, with a 5 th (95th) percentile value of $(-17 \%) 58 \%$. Only seven country pairs exhibit $\mathrm{RSI}^{C}$ above $50 \%$.

Building on the above discussions, we consider the following algorithm to identify $\Theta$.

1. Start with a benchmark close to complete markets (or $m_{t+1}\left(\frac{S_{t+1}}{S_{t}}\right)-m_{t+1}^{\star}=0$ ) and a trial value of $\Theta=0.01$. Solve Problem 1. The output is $\left(\boldsymbol{\lambda}, \delta, d, d^{*}\right)$ and, hence, $m$ and $m^{*}$.

2. Compute the RSI based on the asset market view in equation (28) and compare it to the consumption-based $\mathrm{RSI}^{C}$ in equation (51).

3. Iterate over the choice of $\Theta$ to minimize the discrepancy between the observed consumptionbased risk sharing and the corresponding one from asset returns.

The proposed methodology to identify $\Theta$ for country-specific pairs is, in part, an acknowledgement that $\Theta$ cannot be directly computed from asset return data alone, unless a stand is taken on the size of the unspanned components (i.e., $\operatorname{Var}[u] / \operatorname{Var}[m]$ or $\operatorname{Var}\left[u^{*}\right] / \operatorname{Var}\left[m^{*}\right]$ ). Hampering identification from the mean equation, we further note from equation (47) that $\Theta \mathbb{E}_{t}\left[\delta \sqrt{S_{t+1} / S_{t}}\right]=0$.

In the spirit of Hansen and Jagannathan (1991), we inquire whether the volatilities of the SDF pairs $\left(m_{t+1}, m_{t+1}^{*}\right)$, synthesized using the $\Theta$ estimates, are plausible, while being consistent with the empirical regularity of low consumption risk sharing, and consistent with a lower bound on 
the covariance between the SDFs. Next, we compute the associated SDF correlations, and then investigate if we can address international finance puzzles in our setting of incomplete markets.

In so doing, we strive to bridge some realities of international consumption and asset return data and yet learn about the structure of supportable SDF pairs $\left(m_{t+1}, m_{t+1}^{*}\right)$.

\subsubsection{Discussion and rationale for the estimates of $\Theta$}

Table 1 presents a snapshot of the $\Theta$ estimates across all 45 country pairs, when $\mathbf{R}_{t+1}\left(\mathbf{R}_{t+1}^{*}\right)$ contains six or four assets. The latter is a restricted version that omits the U.S. 30-year Treasury bond and the MSCI world equity index by employing only the risk-free bonds and the equity indices specific to a country pair.

Complementing this evidence, we first investigate whether the $45 \Theta$ estimates obtained with six assets closely correspond, in a statistical sense using the non-parametric Wilcoxon-Mann-Whitney test statistic, to the counterparts with four assets. Our evidence reveals that the associated twosided $p$-value is 0.26 , indicating that we cannot reject that the $\Theta$ estimates have the same underlying distribution. Hence, we focus on the results obtained with six assets in $\mathbf{R}_{t+1}\left(\mathbf{R}_{t+1}^{*}\right)$.

With our procedure and six assets, we observe values of $\Theta$ that have an average 0.70 , a standard deviation of 0.18 , and a 5 th $(95 \mathrm{th})$ percentile value of 0.47 (0.99). We further note from equation (49) that $\Theta>0$ implies that $\operatorname{Var}\left[m_{t+1}\right]>\operatorname{Var}\left[m_{\mathbf{z}, t+1}\right]$, where the latter represents the Hansen and Jagannathan (1991) lower volatility bound with nonnegativity.

Table 2 presents our estimates of $\Theta$ across each of the 45 country pairs. To establish the reported 95\% lower and upper confidence intervals on $\Theta$ displayed in square brackets, we randomly select, with replacement, raw asset returns and recompute $\mathbf{Z}$. We solve again for $\boldsymbol{\lambda}$ via (39), $\delta$ via (40), and then $y_{\mathbf{z}}$. Then, we recompute $m$ and $m^{*}$ and recompute the RSI in equation (45). Finally, we recompute $\Theta$ using the algorithm in Section 3.1.1. We perform 5,000 bootstrap trials.

A particular observation is that our procedure is not tilted toward either low or high values of $\Theta$ with considerable cross-sectional dispersion. Moreover, the average value of $\Theta$ is statistically

distinct from zero. We reach this conclusion from two perspectives. First, regressing the 45 values of $\Theta$ onto a constant yields a two-sided $p$-value of 0.00 . Next, the two-sided $p$-values from a $t$-test, allowing for unequal variance, favors the same conclusion. The solution of $\Theta=0$ is never attained, 
attesting that the market incompleteness parameter is determined by the interplay between the properties of the asset return moments and data on consumption risk sharing.

Some other patterns emerge from Table 2. For example, CAD/USD has the lowest $\Theta$ of 0.39 , while NZD/STG, STG/USD, and FRA/GER manifest below average $\Theta$ estimates of $0.45,0.47$ and 0.57 , respectively. On the other hand, there are some country pairs that manifest above average $\Theta$ estimates, notably, those involving high interest rate differentials, and where one of the countries is Australia or New Zealand. For example, AUD/GER, AUD/JPY, AUD/SWI, NZD/GER, NZD/NLG, and NZD/SWI all exhibit $\Theta$ values exceeding 0.84 .

How sensible are the values of $\Theta$ that minimize the discrepancy between consumption-based risk sharing and the corresponding one from asset returns? To offer a perspective, we set $q=\Theta$ in equations (24) and (25), then $\mathrm{EP}=\frac{1}{2} \Theta \mathbb{E}\left[\boldsymbol{v}^{\prime} \mathbf{Z}\right]$ and $\operatorname{Var}[X]=\operatorname{Var}\left[\frac{1}{2} \Theta \boldsymbol{v}^{\prime} \mathbf{Z}\right]+1=1+\frac{1}{4} \Theta^{2} \operatorname{Var}\left[\boldsymbol{v}^{\prime} \mathbf{Z}\right]$. If we use benchmark values of an excess return of 0.08 and standard deviation of 0.16 , then $\mathbb{E}\left[\boldsymbol{v}^{\prime} \mathbf{Z}\right]=$ 1.08 and $\operatorname{Var}\left[\boldsymbol{v}^{\prime} \mathbf{Z}\right]=0.16^{2}$. Then a value of, for example, $\Theta=0.70$, the average in Panel A of Table 1, (respectively, $\Theta=0.47$ (5th percentile) or $\Theta=0.99$ (95th percentile)) results in a rewardfor-risk $\mathrm{EP} / \sqrt{\operatorname{Var}[X]} \approx 0.38$ (respectively, $\approx 0.25$ (5th percentile), or $\approx 0.53$ (95th percentile) .

Ruling out "good deals," associated with a reward-for-risk $\mathrm{EP} / \sqrt{\operatorname{Var}[X]} \approx 0.38$, on average, lowers the risk sharing index, implied from asset return data, sufficiently to be able to align it with that computed from consumption growth data. Put differently, one can align the observed consumption-based risk sharing and the corresponding one from asset returns, on average, across the 45 country pairs, if one believes that there might be sufficient divergence between the valuations of securities outside the linear span of $\mathbf{R}$ ( or $\mathbf{R}^{\star}$ ) that a reward-for-risk of 0.38 is possible.

Cochrane and Saá-Requejo (2000, page 82) suggest eliminating good deals by ruling out Sharpe ratios greater than twice the Sharpe ratio available on a broad-based equity index. Their choice is not directly observable, but is instead based on introspection and common sense. Still, in search of another benchmark, we bridge implementation and theory by considering empirical analogs. We compute the Appraisal Ratio as the ratio of the mean to standard deviation of foreign equity index returns over and above domestic equity index returns across all currency pairs. The most favorable annualized Appraisal Ratio is 0.26 (NLG over and above JPY). Twice this figure is 0.52. 
The message is that there exist values of $\Theta$ not equal to zero, such that one can reconcile the evidence on consumption-based risk sharing with that from asset returns. Further, the values of $\Theta$ are, from several different angles, economically motivated and anchored around sensible benchmarks.

\subsection{Gauging empirical plausibility: Volatilities and correlations among SDFs}

One may surmise that non-zero values of $\Theta$ will increase the volatility of $m$ and $m^{\star}$. Thus, an important question is: Are the SDFs unrealistically volatile in our incomplete markets setting? An equally relevant question is: What are the derived magnitudes of the correlation between the SDFs. Finally, can our modeling of $\left(m_{t+1}, m_{t+1}^{*}\right)$ pairs help to improve the understanding of economic phenomena, as seen from the vantage point of international finance puzzles?

Our closed-form tractability enables us to provide quantitative guidance on the size of the country-specific unspanned components, computed as $\frac{\operatorname{Var}[u]}{\operatorname{Var}[m]}$ and $\frac{\operatorname{Var}\left[u^{*}\right]}{\operatorname{Var}\left[m^{*}\right]}$, thus, taking us beyond Hansen and Jagannathan (1991), who provide insights about the spanned components of the SDFs.

To get a start on addressing some of the aforementioned questions, we compute the volatilities of $m$ and $m^{*}$, denoted by $\sigma[m]$ and $\sigma\left[m^{*}\right]$, together with the pairwise correlation between $m$ and $m^{*}$, denoted by $\rho_{m, m^{*}}$. The results are reported in Table 2 , and the snapshot is in Table 3 .

In order to interpret these numbers, we consider the conceptually important benchmark of $\Theta=0.01$, which corresponds to a situation in which $\frac{\operatorname{Var}[u]}{\operatorname{Var}[m]}$ and $\frac{\operatorname{Var}\left[u^{*}\right]}{\operatorname{Var}\left[m^{*}\right]}$ are virtually zero.

\begin{tabular}{cccccccccc}
\hline & Avg. & SD & Min. & Max. & 5 th & 25 th & 50 th & 75 th & 95 th \\
\hline$\left.\sigma[m]\right|_{\Theta=0.01}$ & 43 & 7 & 24 & 53 & 33 & 37 & 44 & 49 & 51 \\
$\left.\sigma\left[m^{*}\right]\right|_{\Theta=0.01}$ & 45 & 6 & 30 & 54 & 36 & 39 & 45 & 50 & 52 \\
$\left.\rho_{m, m^{*}}\right|_{\Theta=0.01}$ & 0.96 & 0.02 & 0.86 & 1.0 & 0.93 & 0.95 & 0.97 & 0.98 & 1.00 \\
\hline
\end{tabular}

The values of $\left.\rho_{m, m^{*}}\right|_{\Theta=0.01}$ are between 0.86 and 1.00, establishing that, in the absence of an unspanned component of SDFs, the SDF correlations across the 45 pairs are universally high.

Our quantitative evaluation, reported in Tables 2 and 3, zeros in on two aspects of the economic environment. First, using the estimated values of $\Theta$ increases the volatility of $m$ and $m^{\star}$, enabling concrete insights about the nature of the unspanned component of SDFs that are supportable in our system of incomplete markets. 
Second, our analysis quantifies the most pessimistic values of $\rho_{m, m^{*}}$ in incomplete economies that are compatible with the lower bound on $\operatorname{Cov}\left[m, m^{*}\right]$. In particular, the decline in $\rho_{m, m^{*}}$ is pronounced, consistent with $\operatorname{Cov}\left[u, u^{*}\right]$ becoming more negative. The average $\rho_{m, m^{*}}$ is 0.26 (the 5 th and 95 th percentile value is -0.16 and 0.58 , respectively), which contrasts the average value of 0.96 (the 5 th and 95 th percentile value is 0.93 and 1.00 , respectively) when $\Theta=0.01$.

Our exercises elicit the observation that the size of $\frac{\operatorname{Var}[u]}{\operatorname{Var}[m]}$ has a minimum value of 0.21 and a maximum value of 0.65 , with an average of 0.38 (as presented in Panel $\mathrm{C}$ of Table 3). Providing an additional feel for the importance of the unspanned components among our sample of countries, the average size of the unspanned component for the United States, measured against the other nine industrialized countries, is 0.28 and ranges between 0.18 to 0.38 .

The takeaway is that relaxing $\Theta=0$ (or, allowing $m\left(\frac{S_{t+1}}{S_{t}}\right)-m^{\star} \neq 0$ ), translates into less correlated $m$ and $m^{\star}$ pairs and sizable $\frac{\operatorname{Var}[u]}{\operatorname{Var}[m]}$ and $\frac{\operatorname{Var}\left[u^{*}\right]}{\operatorname{Var}\left[m^{*}\right]}$, culminating into a consistency between the risk sharing index from consumption and asset return data.

Expanding on these themes, the average values of $\sigma[m]$ and $\sigma\left[m^{*}\right]$ (consistent with our lower bound on covariances), across the 45 country pairs, are $57 \%$ and $60 \%$. These values are higher than the corresponding values when $\Theta=0.01$ - as they must be (by equation (49)) - but the new insight is that none of the SDF volatilities is so high as to be implausible. Specifically, the reported values of $\sigma[m]$ and $\sigma\left[m^{*}\right]$ are not out of line with textbook benchmarks (e.g., Cochrane (2005, page 456)).

We consider two exercises that establish robustness:

Robustness Exercise A: What is the impact of expanding assets in $\mathbf{R}_{t+1}\left(\mathbf{R}_{t+1}^{*}\right)$ ? We experimented with several choices, and our conclusions remain robust. For example, we increased the dimensionality of $\mathbf{R}_{t+1}\left(\mathbf{R}_{t+1}^{*}\right)$ to seven by augmenting the set of asset returns to include that of the S\&P commodity index. At the heart of our finding, as seen from Table Internet-II, is that the average $\Theta$ estimated is 0.72 , the average $\frac{\operatorname{Var}[u]}{\operatorname{Var}[m]}$ is 0.40 , with almost no effect on the average $\rho_{m, m^{*}}$. Robustness Exercise B: Instead of estimating values of $\Theta$, necessarily different for each country pair, we ask: Is there a value of $\Theta$, common across the industrialized countries, at which the risk sharing from consumption most closely corresponds, in a statistical sense using the non-parametric Wilcoxon-Mann-Whitney test statistic, to the counterparts from asset returns? The associated two-sided $p$-value with $\Theta=0.60$ (respectively, $\Theta=0.65$ ) is 0.32 (respectively, 0.58), indicating 
that we cannot reject that risk sharing extracted from consumption, and asset returns have the same underlying distribution. The two-sided $p$-values for other values of $\Theta$ are all below 0.05 . Comparing Table Internet-III and Table 3 speaks to the consistency of our conclusions.

Closing, our theoretical and empirical results contain two messages for enriching international finance models. First, our depiction of international consumption and asset market data calls for modeling the composition of the SDFs, and in particular, incorporating nontrivial unspanned components. Second, our exercises suggest that the correlations between domestic and foreign SDFs (and, thus, international risk sharing) need not be high.

\subsection{Framing of international finance puzzles in incomplete markets}

This section considers the correspondence between incomplete markets and three puzzles of complete markets: (i) the forward premium puzzle, (ii) the Backus and Smith puzzle, and (iii) the volatility/risk sharing puzzle. Our interest lies in exploring whether these puzzles are amenable to reconciliation through a route in which markets are deemed to be incomplete.

\subsubsection{Incomplete markets and expected excess return of foreign exchange}

How do deviations from market completeness, $\Theta$, affect the expected excess return of foreign exchange (i.e., the currency risk premium)? The answer in our framework is: There is no effect.

Intuitively, raising $\Theta$ increases the contribution of the zero-mean unspanned components $u$ and $u^{*}$, which should not, and does not, warrant any additional compensation. Now we prove:

Result 1 Deviations from market completeness, $\Theta$, are irrelevant for the expected excess return of foreign exchange, given by

$$
\mathbb{E}_{t}\left[\mathrm{er}_{t+1}^{\mathrm{fx}}\right] \equiv \underbrace{\mathbb{E}_{t}\left[\frac{S_{t+1}}{S_{t}}\right]-\frac{F_{t}}{S_{t}}}_{\mathbb{E}_{t}\left[1+r_{t+1}^{\mathrm{fx}}\right]-R_{f} / R_{f}^{*}}=-R_{f} \operatorname{Cov}_{t}\left[m_{\mathbf{z}, t+1}, r_{t+1}^{\mathrm{fx}}\right], \quad \text { where } r_{t+1}^{\mathrm{fx}} \equiv \frac{S_{t+1}}{S_{t}}-1
$$

and $F_{t} \equiv S_{t} R_{f} / R_{f}^{*}$ is the forward exchange rate.

Proof: We know from the pricing of the foreign currency risk-free return $\left(S_{t+1} / S_{t}\right) R_{f}^{*}$ that $\mathbb{E}_{t}\left[m_{t+1}\left(S_{t+1} / S_{t}\right) R_{f}^{*}\right]=1$. Exploiting $m_{t+1}=m_{\mathbf{z}, t+1}+u_{t+1}$, and using the orthogonality con- 
dition $\mathbb{E}_{t}\left[\delta \sqrt{S_{t+1} / S_{t}}\right]=0$ in equation (48), we deduce $\mathbb{E}_{t}\left[m_{\mathbf{z}, t+1}\left(S_{t+1} / S_{t}\right)\right]=1 / R_{f}^{*}$. Then, using the definition of covariance implies $\mathbb{E}_{t}\left[1+r_{t+1}^{\mathrm{fx}}\right]=R_{f} / R_{f}^{*}-R_{f} \operatorname{Cov}_{t}\left[m_{\mathbf{z}, t+1}, r_{t+1}^{\mathrm{fx}}\right]$.

Result 1 is enormously important. For any value of $\Theta$ whatsoever, our framework is automatically compatible with the expected excess return of foreign exchange.

It is worth emphasizing that our Result 1 is fundamentally different from Lustig and Verdelhan (2016, Corollary 2), who argue that currency risk premiums are changed by the multiplicative wedge perturbation under incomplete spanning. Ongoing questions about the validity of this result are also the subject of Bakshi and Crosby (2016, Claim 1). They show that in the multiplicative wedge

approach (in which $m_{t+1}\left(\frac{S_{t+1}}{S_{t}}\right)=m_{t+1}^{*} e^{\eta_{t+1}}$ ), the currency risk premium does not depend on the multiplicative wedge perturbation $\eta_{t+1}$. Thus, our Result 1 is not specific to models of incomplete markets that give rise to additive form of the SDFs with spanned and unspanned components.

\subsubsection{Incomplete markets and the Backus and Smith (1993) puzzle}

Predicating on a two-country complete markets economy with non-traded goods, the theory of Backus and Smith (1993, Proposition 2, equation (4.8)) restricts the slope coefficient to unity when (log) changes in real exchange rates are regressed on relative (log) marginal utility growth, or equivalently, $(\log )$ relative SDFs. The puzzle is the lack of empirical support for the hypothesized relationship, gauged by the regression slope coefficients that are substantially different from unity.

How to interpret Backus and Smith (1993) puzzle in incomplete markets? We do so from two perspectives. Departing from complete markets, we have endeavored to construct $\left(m_{t+1}, m_{t+1}^{*}\right)$ pairs by solving an optimization problem that minimizes the covariance between the SDFs, which rules out trades with unacceptably large reward-for-risk, which enforces correct pricing and the nonnegativity of SDFs, while being also consistent with limited risk sharing. The null hypothesis of complete markets then translates into an empirical testing equation with identifiable restrictions:

$$
\begin{array}{r}
\log \left(S_{t+1} / S_{t}\right)=\Pi_{0}+\Pi_{1} \log \left(m_{t+1}^{*} / m_{t+1}\right)+e_{t+1}, \\
\text { with the null hypothesis : } \Pi_{0}=0 \text { and } \Pi_{1}=1 .
\end{array}
$$

The population regression coefficient $\Pi_{1}=\operatorname{Cov}\left(\log \left(S_{t+1} / S_{t}\right), \log \left(m_{t+1}^{*} / m_{t+1}\right)\right) / \operatorname{Var}\left(\log \left(m_{t+1}^{*} / m_{t+1}\right)\right)$ is not analytical, but can be computed based on the constructed $\left(m_{t+1}, m_{t+1}^{*}\right)$ pairs and the ob- 
served $\log \left(S_{t+1} / S_{t}\right)$. Table 4 presents our findings, focusing on country pairs in which the domestic country is either the United States, Japan, or Switzerland (for space considerations).

Our results show that there is a relationship between the real exchange rate growth and the $\left(m_{t+1}, m_{t+1}^{*}\right)$ pairs but it is not one-for-one. The $\Pi_{1}$ coefficients range between -0.00 and 0.08 , and the hypothesis of $\Pi_{1}=1$ is overwhelmingly rejected based on the $p$-values from the Wald test. The documented deviations of $\Pi_{1}$ from unity in our incomplete markets setting is indicative of the prevailing empirical evidence on the Backus and Smith regressions.

The second perspective is that incomplete markets imply a multitude of SDFs. In this case, equation (5) implies $\mathbb{E}_{t}\left[\xi_{t+1}\right]=0, \mathbb{E}_{t}\left[\xi_{t+1} \mathbf{R}_{t+1}^{\star}\right]=0, \mathbb{E}_{t}\left[\xi_{t+1}^{*}\right]=0$, and $\mathbb{E}_{t}\left[\xi_{t+1}^{*} \mathbf{R}_{t+1}\right]=0$. This prompts us to formulate a hypothesis about $\left(m_{t+1}, m_{t+1}^{*}\right)$ pairs and exchange rate growth as

$$
m_{t+1}\left(\frac{S_{t+1}}{S_{t}}\right)-m_{t+1}^{\star}=\psi_{0}+\varepsilon_{t+1}, \quad m_{t+1}-m_{t+1}^{\star} /\left(\frac{S_{t+1}}{S_{t}}\right)=\psi_{0}^{*}+\varepsilon_{t+1}^{*}
$$

where $\left(\varepsilon_{t+1}, \varepsilon_{t+1}^{*}\right)$ are zero-mean regression residuals. The testable hypothesis is $\psi_{0}=0$ and $\psi_{0}^{*}=0$.

One may view (54) as the analogue to Backus and Smith (1993, Proposition 2) in incomplete markets, but with the testing equation rearranged and in non-log form.

In moving from theory to implementation, we cast (54) as a SUR estimation. When $\left(m_{t+1}, m_{t+1}^{*}\right)$ are based on the country-specific $\Theta$ estimates, the $\psi_{0}$ and $\psi_{0}^{*}$ estimates are uniformly close to zero and individually and jointly insignificant. These estimates are not reported to save on space.

So what is the bottom line? In line with Backus and Smith (1993), we have tested relations that

link the moments of the triplet $\left(m_{t+1}, m_{t+1}^{*}, \frac{S_{t+1}}{S_{t}}\right)$. The first one in (53) is empirically inspired, while the other one in (54) is exact, but in expectations form. Our estimation results offer a contrast to Lustig and Verdelhan (2016, Section 2.3), who are agnostic on whether low slope coefficients in the Backus and Smith regressions can be obtained through the mechanism of incomplete markets.

\subsubsection{Reinterpretation of the volatility/risk sharing puzzle in incomplete markets}

The volatility/risk sharing puzzle, as framed and exposed in Brandt, Cochrane, and Santa-Clara (2006), is that when $\log \left(\frac{S_{t+1}}{S_{t}}\right)=\log \left(m_{t+1}^{\star}\right)-\log \left(m_{t+1}\right)$ holds state-by-state in complete markets, 
one can rationalize the relatively low volatility of (log) exchange rate growth observed in the data only if $\log \left(m_{t+1}\right)$ and $\log \left(m_{t+1}^{*}\right)$ are highly correlated, or if the risk sharing index in $(28)$ is high.

Matters are conceptually different in incomplete markets. Some SDFs satisfy $m_{t+1}\left(\frac{S_{t+1}}{S_{t}}\right)-$ $m_{t+1}^{*}=0$ or $m_{t+1}-m_{t+1}^{*} /\left(\frac{S_{t+1}}{S_{t}}\right)=0$, while others do not. In particular, when $\mathbb{E}_{t}\left[\xi_{t+1}\right]=0$, or $\mathbb{E}_{t}\left[m_{t+1}\left(\frac{S_{t+1}}{S_{t}}\right)-m_{t+1}^{\star}\right]=0$, there is no longer a tractable functional relationship that allows one to take logs and apply the operations of expectation or variances. Instead, we take the exchange rate dynamics, along with other asset returns, as fixed by the data, and solve for the SDFs. Thus, there is no unique mapping between $m_{t+1}, m_{t+1}^{*}$, and $\frac{S_{t+1}}{S_{t}}$ in incomplete markets, suggesting that the volatility puzzle may not be well posed.

Elaborating on the above, we note that $\mathbb{E}_{t}\left[\xi_{t+1}\right]=0$ and $\mathbb{E}_{t}\left[\xi_{t+1}^{*}\right]=0$ always hold, regardless of whether the market is complete or incomplete. For instance, $\mathbb{E}_{t}\left[\xi_{t+1}\right]=0$ is isomorphic to $\frac{F_{t}}{S_{t}}=$ $\frac{\mathbb{E}_{t}\left[m_{t+1}^{*}\right]}{\overline{\mathbb{E}_{t}}\left[m_{t+1}\right]}$, whereas $\mathbb{E}_{t}\left[\xi_{t+1}^{*}\right]=0$ is isomorphic to $\frac{F_{t}^{-1}}{S_{t}^{-1}}=\frac{\mathbb{E}_{t}\left[m_{t+1}\right]}{\mathbb{E}_{t}\left[m_{t+1}^{*}\right]}$. These restrictions are equivalent to testing $\Psi_{0}=\Psi_{0}^{*}=0$ (as outlined in equation (54)) and are not rejected with our synthesized $\left(m_{t+1}, m_{t+1}^{*}\right)$. The essential point is that the SDFs are consistent with the time series of $\left(\frac{S_{t+1}}{S_{t}}\right)$.

To examine empirical plausibility, and at the same time comment on the consistency of our approach with the volatility of exchange rate growth, we propose a result that holds under all martingale measures, irrespective of whether markets are complete or incomplete. Now we state:

Result 2 Each $m_{t+1}$ satisfies

$$
\mathbb{E}_{t}\left[\left(r_{t+1}^{\mathrm{fx}}-\mathbb{E}_{t}\left[r_{t+1}^{\mathrm{fx}}\right]\right)^{2}\right]=\operatorname{vrp}_{t+1}^{\mathrm{fx}}+\underbrace{\mathbb{E}_{t}\left[\frac{m_{t+1}}{\mathbb{E}_{t}\left[m_{t+1}\right]}\left(r_{t+1}^{\mathrm{fx}}\right)^{2}\right]-\left(\frac{F_{t}}{S_{t}}-1\right)^{2}}_{\text {risk-neutral currency variance }}
$$

where $\operatorname{vrp}_{t+1}^{\mathrm{fx}} \equiv-\operatorname{Cov}_{t}\left[\frac{m_{t+1}}{\mathbb{E}_{t}\left[m_{t+1}\right]},\left(r_{t+1}^{\mathrm{fx}}-\mathbb{E}_{t}\left[r_{t+1}^{\mathrm{fx}}\right]\right)^{2}\right]+\left(\mathbb{E}_{t}\left[r_{t+1}^{\mathrm{fx}}\right]-\left(\frac{F_{t}}{S_{t}}-1\right)\right)^{2}$ is the currency variance risk premium. Moreover, deviations from market completeness, $\Theta$, can be relevant for $\operatorname{vrp}_{t+1}^{\mathrm{fx}}$ whenever $\operatorname{Cov}_{t}\left[u_{t+1},\left(r_{t+1}^{\mathrm{fx}}-\mathbb{E}_{t}\left[r_{t+1}^{\mathrm{fx}}\right]\right)^{2}\right]=\frac{1}{2} d \Theta \mathbb{E}_{t}\left[\delta\left(S_{t+1} / S_{t}\right)^{3 / 2}\right] \neq 0$.

\section{Proof: See Appendix B.}

We can convert equation (55) into a testing equation by considering the cross-sectional regression $v p_{n}=\Omega_{0}+\Omega_{1} v q_{n}+e_{n}$, for $n=1, \ldots, \mathcal{N}$, where the variance $v p^{2} \equiv \mathbb{E}\left[\left(r_{t+1}^{\mathrm{fx}}-\mathbb{E}\left[r_{t+1}^{\mathrm{fx}}\right]\right)^{2}\right]$ is computed from the time series of exchange rate growth. With $m_{t+1}$ synthesized from Problem 1, we compute 
the model risk-neutral variance $v q^{2} \equiv \mathbb{E}\left[\frac{m_{t+1}}{\mathbb{E}\left[m_{t+1}\right]}\left(r_{t+1}^{\mathrm{fx}}\right)^{2}\right]-\left(\mathbb{E}\left[\frac{m_{t+1}}{\mathbb{E}\left[m_{t+1}\right]} r_{t+1}^{\mathrm{fx}}\right]\right)^{2}$ and report the OLS regression results with standard errors corrected for heteroscedasticity (for each of USD, JPY, and SWI as the domestic currency against the remaining nine currencies following Table 4):

\begin{tabular}{ccccccccc}
\hline & \multicolumn{2}{c}{$\Omega_{0}$} & & \multicolumn{2}{c}{$\Omega_{1}$} & & \multicolumn{2}{c}{ Wald $p$-val. } \\
\cline { 2 - 3 } & coeff. & $p$-val. & & coeff. & $p$-val. & & $\Omega_{1}=1$ & $\mathcal{N}$ \\
\hline All 27 pairs & -0.114 & 0.037 & & 1.009 & 0.000 & & 0.083 & 27 \\
\hline
\end{tabular}

Two conclusions can be drawn from our analysis. First, as reflected in $\Omega_{1}$ close to unity, the average risk-neutral currency volatilities generated in our incomplete market setting (and Problem 1) appear anchored to the average unconditional currency volatilities. Second, the finding $\Omega_{0}<0$ points to the presence of a negative (average) currency variance risk premium. The latter is consistent with the evidence from currency options markets (e.g., Ammann and Buesser (2013, Table 1)).

Closing, we have shown that the most pessimistic estimates of correlations between the domestic and foreign SDFs need not be high. This attribute of our analysis in incomplete markets counteracts the intuition that SDFs, implied by asset return data when $m_{t+1}\left(\frac{S_{t+1}}{S_{t}}\right)-m_{t+1}^{*}=0$ is assumed, must be highly correlated to reproduce the low volatility of (log) exchange rate growth. Moreover, the SDFs constructed from Problem 1 point to negative (average) currency variance risk premiums, even though we did not incorporate currency options return data in their construction.

\section{Conclusions}

We present a framework for characterizing domestic and foreign SDF pairs in international economies and incomplete markets, with novel ingredients. Importantly, we do not assume that exchange rate growth equals the ratio of SDFs. Moreover, we develop a restriction that precludes "good deals" in international economies with incomplete markets. We show that ruling out "good deals - the possibility to form a portfolio with an implausibly high reward-for-risk - places an upper bound on the dispersion of the domestic and foreign SDFs.

A derived feature of our model is an additive form of the SDFs in which the SDFs are analytically decomposed into their spanned and unspanned components, and a low correlation between 
the domestic and foreign SDFs can arise when the country-specific unspanned components are negatively correlated. We show that our solution, with an additive form of the SDFs, lies outside of the class of SDFs that are possible under the multiplicative wedge approach of Backus, Foresi, and Telmer (2001) and considered further in Lustig and Verdelhan (2016).

At the core of our analysis is the market incompleteness parameter that quantifies the upper bound on the dispersion of the SDFs through its role as the maximum reward-for-risk. We consider an algorithm to estimate the market incompleteness parameter from consumption growth data, in conjunction with asset return data. The resulting framework of incomplete markets is both tractable and versatile: It can be aligned to patterns of limited international risk sharing, can accommodate a relatively large difference between the unspanned components of the SDFs, and can realistically model the volatility of SDFs and their correlations.

Additionally, we show that our incomplete markets approach is useful for thinking about international finance puzzles. Considering first the forward premium puzzle, our framework, through the channel of the spanned components of the SDFs, reproduces a currency risk premium consistent with the data. Going further, our model of incomplete markets remedies shortcomings of complete market models with respect to the Backus and Smith (1993) puzzle: we show that a regression of log exchange rate growth on log relative SDFs constructed from our model generate slope coefficients (and correlations) in line with observable patterns. Finally, we show that it is possible to generate low correlations between the domestic and foreign SDFs. This feature of our model merits attention in the context of the volatility/risk sharing puzzle, and offers a source of differentiation from Brandt, Cochrane, and Santa-Clara (2006), who argue that the correlations between the SDFs and the level of international risk sharing, imputed from asset return data, must be high.

This paper has provided an improved and economically motivated framework for modeling SDFs, and their correlations, in a system of incomplete international economies. The approach is consistent with the evidence on limited international risk sharing. 


\section{References}

M. Ammann and R. Buesser. Variance risk premiums in foreign exchange markets. Journal of Empirical Finance, 23:16-32, 2013.

D. Backus and G. Smith. Consumption and real exchange rates in dynamic economies with nontraded goods. Journal of International Economics, 35:297-316, 1993.

D. Backus, S. Foresi, and C. Telmer. Affine term structure models and the forward premium anomaly. Journal of Finance, 56(1):279-304, 2001.

G. Bakshi and J. Crosby. The multiplicative wedge approach to incomplete markets does not resolve the trifecta of exchange rate puzzles. Unpublished working paper (http://ssrn.com/abstract=2837461), University of Maryland, 2016.

N. Balakrishnan and C. Lai. Continuous Bivariate Distributions. Springer, New York, NY, 2009.

R. Barro and J. Ursua. Macroeconomic crises since 1870. Brookings Papers on Economic Activity, 2008.

M. Brandt, J. Cochrane, and P. Santa-Clara. International risk sharing is better than you think, or exchange rates are too smooth. Journal of Monetary Economics, 53:671-698, 2006.

C. Burnside and J. Graveline. Exchange rate determination, risk sharing and the asset market view. Unpublished working paper, NBER \# 18646, 2014.

J. Cochrane. Asset Pricing. Princeton University Press, 2005.

J. Cochrane and J. Saá-Requejo. Beyond arbitrage: Good-Deal asset price bounds in incomplete markets. Journal of Political Economy, 108:79-119, 2000.

R. Colacito and M. Croce. Risks for the long run and the real exchange rate. Journal of Political Economy, 119(1):153-181, 2011.

R. Colacito and M. Croce. International asset pricing with recursive preferences. Journal of Finance, 68(6):2651-2686, 2013. 
R. Colacito, M. Croce, F. Gavazzoni, and R. Ready. Currency risk factors in a recursive multicountry economy. Unpublished working paper (http://ssrn.com/abstract=2542182), University of North Carolina, INSEAD, and University of Rochester, 2015.

G. Corsetti, L. Dedola, and S. Leduc. International risk sharing and the transmission of productivity shocks. Review of Economic Studies, 75:443-473, 2008.

D. Duffie. Dynamic Asset Pricing Theory. Cambridge Publishing, Cambrige, 1992.

E. Fama. Forward and spot exchange rates. Journal of Monetary Economics, 14:319-338, 1984.

J. Favilukis, L. Garlappi, and S. Neamati. The carry trade and uncovered interest parity when markets are incomplete. Working paper (http://ssrn.com/abstract=2609151), UBC, 2015.

X. Gabaix and M. Maggiori. International liquidity and exchange rate dynamics. Quarterly Journal of Economics, 130:1369-1420, 2015.

F. Gavazzoni, B. Sambalaibat, and C. Telmer. Currency risk and pricing kernel volatility. Unpublished working paper (http://ssrn.com/abstract=2179424), Carnegie Mellon University, 2013.

R. Grinold and R. Kahn. Active Portfolio Management. McGraw Hill, New York, 2007.

L. Hansen and R. Hodrick. Forward exchange rates as optimal predictors of future spot rates: An econometric analysis. Journal of Political Economy, 88:829-853, 1980.

L. Hansen and R. Jagannathan. Implications of security market data for models of dynamic economies. Journal of Political Economy, 99:225-262, 1991.

N. Kim and A. Schiller. Limited stock market participation and goods market frictions: A potential resolution for puzzles in international finance. Unpublished working paper (http://www.andrew.cmu.edu/user/aschille/limitedparticipationinternational.pdf), Carnegie Mellon University, 2015.

K. Lewis. What can explain the lack of international consumption risk sharing? Journal of Political Economy, 104:267-297, 1996.

K. Lewis and E. Liu. Evaluating international consumption risk sharing gains: An asset return view. Journal of Monetary Economics, 71:84-98, 2015. 
H. Lustig and A. Verdelhan. Does incomplete spanning in international financial markets help to explain exchange rates? NBER working paper \# 22023 (June 20, 2016, https://westernfinanceportal.org/viewpaper.php?n=446544), Stanford and MIT, 2016.

T. Maurer and N.-K. Tran. Entangled risks in incomplete FX markets. Unpublished working paper (http://ssrn.com/abstract=2697278), University of Washington, St. Louis, 2015.

W. Newey and K. West. A simple, positive semi-definite, heteroskedasticity and autocorrelation consistent covariance matrix. Econometrica, 55:703-708, 1987.

W. Newey and K. West. Automatic lag selection in covariance matrix estimation. Review of Economic Studies, 61(4):631-653, 1994.

A. Pavlova and R. Rigobon. Asset prices and exchange rates. Review of Financial Studies, 20: 1139-1180, 2007.

R. Roll. A mean/variance analysis of tracking error. Journal of Portfolio Management, 18:13-22, 1992.

W. Sharpe. Decentralized investment management. Journal of Finance, 36(2):217-234, 1981.

A. Stathopoulos. Asset prices and risk sharing in open economies. Unpublished working paper (http://ssrn.com/abstract=1364082), University of Washington, 2015.

S. Zhang. Limited risk sharing and international equity returns. Unpublished working paper (http://ssrn.com/abstract=2542182), New York University, 2015. 


\section{A Appendix A: $\left|\mathbb{E}\left[y-y^{\star}\right]\right|$ should, in practice, be very small and proof that} $\mathbb{E}\left[y-y^{\star}\right]=0$ when $m, m^{\star}$ and $S_{t+1} / S_{t}$ are lognormally distributed

Our objective is to show that (i) $\left|\mathbb{E}\left[y-y^{\star}\right]\right|$ should, in practice, be very small, and (ii) $\mathbb{E}\left[y-y^{\star}\right]$ is identically zero when $m, m^{\star}$ and $S_{t+1} / S_{t}$ are lognormally distributed.

We focus first on the case in which $\log (m), \log \left(m^{\star}\right)$, and $\log \left(S_{t+1} / S_{t}\right)$ are jointly normally distributed, and prove an exact result. We have

$$
\mathbb{E}[m]=1 / R_{f}, \quad \mathbb{E}\left[m^{\star}\right]=1 / R_{f}^{\star}, \quad \text { and } \quad \mathbb{E}\left[S_{t+1} / S_{t}\right] \equiv \exp \left(\mu_{s}\right),
$$

and the variances are $\operatorname{Var}[\log (m)]$, $\operatorname{Var}\left[\log \left(m^{\star}\right)\right]$, and $\operatorname{Var}\left[\log \left(S_{t+1} / S_{t}\right)\right] \equiv \sigma_{s}^{2}$, respectively.

Using standard results on moment generating functions, $\mathbb{E}\left[\left(S_{t+1} / S_{t}\right)^{1 / 2}\right]=\exp \left(\frac{1}{2} \mu_{s}-\frac{1}{8} \sigma_{s}^{2}\right)$ and $\mathbb{E}\left[\left(S_{t+1} / S_{t}\right)^{-1 / 2}\right]=\exp \left(-\frac{1}{2} \mu_{s}+\frac{3}{8} \sigma_{s}^{2}\right)$. Equation (4), namely, $\mathbb{E}_{t}\left[m\left(\frac{S_{t+1}}{S_{t}}\right)-m^{\star}\right]=0$, implies

$$
\frac{1}{R_{f}^{\star}}=\frac{1}{R_{f}} \exp \left(\mu_{s}+\operatorname{Cov}\left[\log (m), \log \left(S_{t+1} / S_{t}\right)\right]\right),
$$

while $\mathbb{E}\left[m-m^{\star} /\left(\frac{S_{t+1}}{S_{t}}\right)\right]=0$ implies (i.e., Balakrishnan and Lai (2009, equation (11.69), page 526)):

$$
\frac{1}{R_{f}}=\frac{1}{R_{f}^{\star}} \exp \left(-\mu_{s}+\sigma_{s}^{2}-\operatorname{Cov}\left[\log \left(m^{\star}\right), \log \left(S_{t+1} / S_{t}\right)\right]\right) .
$$

Further,

$$
\begin{aligned}
\mathbb{E}[y]=\mathbb{E}\left[m\left(S_{t+1} / S_{t}\right)^{1 / 2}\right] & =\left(1 / R_{f}\right) \exp \left(\frac{1}{2} \mu_{s}+\frac{1}{2} \operatorname{Cov}\left[\log (m), \log \left(S_{t+1} / S_{t}\right)\right]-\frac{1}{8} \sigma_{s}^{2}\right), \\
& =1 / \sqrt{R_{f} R_{f}^{\star}} \exp \left(-\frac{1}{8} \sigma_{s}^{2}\right), \quad(\operatorname{using}(A 2))
\end{aligned}
$$

and also,

$$
\begin{aligned}
\mathbb{E}\left[y^{*}\right]=\mathbb{E}\left[m^{\star}\left(S_{t+1} / S_{t}\right)^{-1 / 2}\right] & =\left(1 / R_{f}^{\star}\right) \exp \left(-\frac{1}{2} \mu_{s}-\frac{1}{2} \operatorname{Cov}\left[\log \left(m^{\star}\right), \log \left(S_{t+1} / S_{t}\right)\right]+\frac{3}{8} \sigma_{s}^{2}\right) \\
& =1 / \sqrt{R_{f} R_{f}^{\star}} \exp \left(-\frac{1}{8} \sigma_{s}^{2}\right), \quad(\text { using }(A 3)),
\end{aligned}
$$

or $\mathbb{E}\left[y-y^{\star}\right]=0$. Our assertion is proved. 
Next, we show $\left|\mathbb{E}\left[y-y^{\star}\right]\right| \approx 0$, in general. With $y=m \sqrt{S_{t+1} / S_{t}}$ and $y^{\star}=m^{\star} / \sqrt{S_{t+1} / S_{t}}$, the analog to equation (4) is

$$
\mathbb{E}\left[\left(y-y^{\star}\right)\left(S_{t+1} / S_{t}\right)^{1 / 2}\right]=0 \quad \text { and, likewise, } \quad \mathbb{E}\left[\left(y-y^{\star}\right) \frac{1}{\left(S_{t+1} / S_{t}\right)^{1 / 2}}\right]=0
$$

More generally, defining $g[\phi] \equiv\left(\phi S_{t+1} / S_{t}\right)^{1 / 2}+1 /\left(\phi S_{t+1} / S_{t}\right)^{1 / 2}$, for any $\phi$, satisfying $0<\phi<$ $+\infty$, we have

$$
\mathbb{E}\left[\left(y-y^{\star}\right)\left(\phi S_{t+1} / S_{t}\right)^{1 / 2}+\left(y-y^{\star}\right) /\left(\phi S_{t+1} / S_{t}\right)^{1 / 2}\right] \equiv \mathbb{E}\left[\left(y-y^{\star}\right) g[\phi]\right]=0 .
$$

Hence, $\operatorname{Cov}\left[y-y^{\star}, g[\phi]\right]=-\mathbb{E}\left[y-y^{\star}\right] \mathbb{E}[g[\phi]]$. Thus, $\left|\mathbb{E}\left[y-y^{\star}\right]\right|^{2}(\mathbb{E}[g[\phi]])^{2} \leq \operatorname{Var}\left[y-y^{\star}\right] \operatorname{Var}[g[\phi]]$. Or,

$$
\left|\mathbb{E}\left[y-y^{\star}\right]\right|^{2}\left\{(\mathbb{E}[g[\phi]])^{2}-\operatorname{Var}[g[\phi]]\right\} \leq \mathbb{E}\left[\left(y-y^{\star}\right)^{2}\right] \operatorname{Var}[g[\phi]]
$$

We further note that $\operatorname{Var}[g[\phi]]=\mathbb{E}\left[\left(\left(\phi S_{t+1} / S_{t}\right)+1 /\left(\phi S_{t+1} / S_{t}\right)\right)-2\right]-\mathbb{E}[g[\phi]-2] \mathbb{E}[g[\phi]+2]$.

In practice, for $\phi \approx 1, \operatorname{Var}[g[\phi]]$ may be very small. For example, empirical data suggests $S_{t+1} / S_{t}$ is close to a martingale, so $\mathbb{E}\left[S_{t+1} / S_{t}\right] \approx 1$. Moreover, $S_{t+1} / S_{t}$ has relatively low volatility, suggesting $\mathbb{E}\left[1 /\left(S_{t+1} / S_{t}\right)\right]$ may also be close to unity. Hence, $\mathbb{E}\left[\left(\left(S_{t+1} / S_{t}\right)+1 /\left(S_{t+1} / S_{t}\right)\right)-2\right] \approx 0$, $\mathbb{E}[g[1]] \approx 2$, and $\mathbb{E}[g[1]-2]=\mathbb{E}\left[\left(S_{t+1} / S_{t}\right)^{1 / 2}+1 /\left(S_{t+1} / S_{t}\right)^{1 / 2}-2\right] \approx 0$, and, thus, Var $[g(1)]$ should typically be close to zero. Hence, in practice, equation (A9), evaluated in the special case of $\phi=1$, should imply a tight bound on $\left|\mathbb{E}\left[y-y^{\star}\right]\right|$.

\section{B Appendix B: Proof of Result 2}

For brevity of equation presentation, let $\mu_{t}^{\mathrm{fx}} \equiv \mathbb{E}_{t}\left[r_{t+1}^{\mathrm{fx}}\right]$, where $r_{t+1}^{\mathrm{fx}} \equiv \frac{S_{t+1}}{S_{t}}-1$.

Define the risk-neutral (martingale) measure such that the expectation $\mathbb{E}_{t}^{Q}[$.$] satisfies$

$$
\begin{aligned}
\mathbb{E}_{t}^{Q}\left[\left(r_{t+1}^{\mathrm{fx}}\right)^{2}\right] & =\mathbb{E}_{t}\left[\frac{m_{t+1}}{\mathbb{E}_{t}\left[m_{t+1}\right]}\left(r_{t+1}^{\mathrm{fx}}\right)^{2}\right], \text { and } \\
\mathbb{E}_{t}^{Q}\left[r_{t+1}^{\mathrm{fx}}\right] & =\mathbb{E}_{t}\left[\frac{m_{t+1}}{\mathbb{E}_{t}\left[m_{t+1}\right]} r_{t+1}^{\mathrm{fx}}\right]=\frac{F_{t}}{S_{t}}-1
\end{aligned}
$$


The risk-neutral mean in equation (B2) follows, since $\mathbb{E}_{t}\left[m_{t+1}\left(\frac{S_{t+1}}{S_{t}}-\frac{F_{t}}{S_{t}}\right)\right]=0$. Using the covariance operator,

$$
\mathbb{E}_{t}\left[m_{t+1}\left(r_{t+1}^{\mathrm{fx}}-\mu_{t}^{\mathrm{fx}}\right)^{2}\right]=\operatorname{Cov}_{t}\left[m_{t+1},\left(r_{t+1}^{\mathrm{fx}}-\mu_{t}^{\mathrm{fx}}\right)^{2}\right]+\mathbb{E}_{t}\left[m_{t+1}\right] \mathbb{E}_{t}\left[\left(r_{t+1}^{\mathrm{fx}}-\mu_{t}^{\mathrm{fx}}\right)^{2}\right]
$$

Rearranging and simplifying,

$$
\mathbb{E}_{t}\left[\left(r_{t+1}^{\mathrm{fx}}-\mu_{t}^{\mathrm{fx}}\right)^{2}\right]-\mathbb{E}_{t}^{Q}\left[\left(r_{t+1}^{\mathrm{fx}}-\mu_{t}^{\mathrm{fx}}\right)^{2}\right]=-\frac{1}{\mathbb{E}_{t}\left[m_{t+1}\right]} \operatorname{Cov}_{t}\left[m_{t+1},\left(r_{t+1}^{\mathrm{fx}}-\mu_{t}^{\mathrm{fx}}\right)^{2}\right]
$$

We may reexpress the left hand side of equation (B4) by adding and subtracting the risk-neutral mean $\frac{F_{t}}{S_{t}}-1$ in the second term as

$\mathbb{E}_{t}\left[\left(r_{t+1}^{\mathrm{fx}}-\mu_{t}^{\mathrm{fx}}\right)^{2}\right]-\mathbb{E}_{t}^{Q}\left[\left(r_{t+1}^{\mathrm{fx}}-\left(\frac{F_{t}}{S_{t}}-1\right)-\left\{\mu_{t}^{\mathrm{fx}}-\left(\frac{F_{t}}{S_{t}}-1\right)\right\}\right)^{2}\right]=-\operatorname{Cov}_{t}\left[\frac{m_{t+1}}{\mathbb{E}_{t}\left[m_{t+1}\right]},\left(r_{t+1}^{\mathrm{fx}}-\mu_{t}^{\mathrm{fx}}\right)^{2}\right]$

The expression for the currency variance risk premium is

$$
\underbrace{\mathbb{E}_{t}\left[\left(r_{t+1}^{\mathrm{fx}}-\mu_{t}^{\mathrm{fx}}\right)^{2}\right]-\mathbb{E}_{t}^{Q}\left[\left(r_{t+1}^{\mathrm{fx}}-\left(\frac{F_{t}}{S_{t}}-1\right)\right)^{2}\right]}=\left\{\mu_{t}^{\mathrm{fx}}-\left(\frac{F_{t}}{S_{t}}-1\right)\right\}^{2}-\operatorname{Cov}_{t}\left[\frac{m_{t+1}}{\mathbb{E}_{t}\left[m_{t+1}\right]},\left(r_{t+1}^{\mathrm{fx}}-\mu_{t}^{\mathrm{fx}}\right)^{2}\right] .
$$

Currency variance risk premium

We have the proof of (55) of Result 2, noting that $\mathbb{E}_{t}^{Q}\left[\left(r_{t+1}^{\mathrm{fx}}-\left(\frac{F_{t}}{S_{t}}-1\right)\right)^{2}\right]=\mathbb{E}_{t}^{Q}\left[\left(r_{t+1}^{\mathrm{fx}}\right)^{2}\right]-\left(\frac{F_{t}}{S_{t}}-1\right)^{2}$.

Additionally, the additive form of the SDF implies $m_{t+1}=m_{\mathbf{z}, t+1}+u_{t+1}$, where $u_{t+1}=$ $\frac{1}{2} d \Theta \delta \frac{1}{\sqrt{S_{t+1} / S_{t}}}$ from equation (43). Moreover, $\left(r_{t+1}^{\mathrm{fx}}-\mu_{t}^{\mathrm{fx}}\right)^{2}=\left(r_{t+1}^{\mathrm{fx}}\right)^{2}+\left(\mu_{t}^{\mathrm{fx}}\right)^{2}-2\left(\mu_{t}^{\mathrm{fx}}\right) r_{t+1}^{\mathrm{fx}}$. Then the currency variance risk premium depends on the market incompleteness parameter $\Theta$ via

$$
\operatorname{Cov}_{t}\left[u_{t+1},\left(r_{t+1}^{\mathrm{fx}}-\mu_{t}^{\mathrm{fx}}\right)^{2}\right]=\frac{1}{2} d \Theta \mathbb{E}_{t}\left[\delta\left(S_{t+1} / S_{t}\right)^{3 / 2}\right]
$$

since $\mathbb{E}_{t}\left[\delta \sqrt{S_{t+1} / S_{t}}\right]=0$. It is already established that the currency risk premium $\mathbb{E}_{t}\left[r_{t+1}^{\mathrm{fx}}\right]-\left(\frac{F_{t}}{S_{t}}-1\right)$ is independent of $\Theta$. 


\section{Table 1: A snapshot of the estimates of market incompleteness parameter $\Theta$}

The estimation of $\Theta$ is performed for each of the 45 country pairs of industrialized countries. The countries in our sample are Australia (AUD), New Zealand (NZD), United Kingdom (STG), France (FRA), Canada (CAD), United States (USD), Netherlands (NLG), Germany (GER), Japan (JPY), and Switzerland (SWI). Reported are the mean, the standard deviation, the minimum, the maximum, and the percentiles of the $\Theta$ estimates. Our estimation algorithm incorporates the argument that there exists a value of $\Theta$ that minimizes the discrepancy between the risk sharing index from consumption and asset returns (as detailed in Section 3.1.1). We consider different sets of asset returns, allowing for multi-currency and multi-country exposures. Consider the Australia versus Japan (AUD/JPY) country pair, where the gross return vector $\mathbf{R}_{t+1}$ contains six assets:

$$
\underbrace{\mathbf{R}_{t+1}}_{6 \times 1}=\left\{\begin{array}{l}
\text { Return of the Australian risk-free bond (in AUD) } \\
\text { Return of the Australian equity index (in AUD) } \\
\text { Return of the Japanese risk-free bond (in AUD) } \\
\text { Return of the Japanese equity index (in AUD) } \\
\text { Return of the U.S. 30-year Treasury bond (in AUD) } \\
\text { Return of the MSCI world equity index (in AUD) }
\end{array}\right.
$$

Symmetrically, $\mathbf{R}_{t+1}^{*}=\mathbf{R}_{t+1} /\left(\frac{S_{t+1}}{S_{t}}\right)$ contains the same set of gross returns denominated in Japanese yen, where $\frac{S_{t+1}}{S_{t}}$ is the exchange rate growth with the Japanese yen as the reference currency. The return vector $\mathbf{R}_{t+1}\left(\mathbf{R}_{t+1}^{*}\right)$ with four assets is a restricted version of that with six assets with returns of (i) the Australian risk-free bond, (ii) the Australian equity index, (iii) the Japanese risk-free bond, and (iv) the Japanese equity index. The sample period considered is January 1975 to June 2014 (474 observations).

Mean SD Min. Max. $\quad$\begin{tabular}{c} 
Percentiles \\
\cline { 3 - 5 }
\end{tabular}

Panel A: The dimensionality of $\mathbf{R}_{t+1}\left(\mathbf{R}_{t+1}^{*}\right)$ is six.

$\begin{array}{llllllllll}\text { Six Assets } & 0.70 & 0.18 & 0.39 & 1.13 & 0.47 & 0.56 & 0.68 & 0.84 & 0.99\end{array}$

Panel B: The dimensionality of $\mathbf{R}_{t+1}\left(\mathbf{R}_{t+1}^{*}\right)$ is four.

$\begin{array}{llllllllll}\text { Four Assets } & 0.66 & 0.17 & 0.39 & 1.10 & 0.43 & 0.54 & 0.60 & 0.73 & 0.94\end{array}$


Table 2: Estimates of $\Theta$, SDF volatilities, correlation between SDFs, and the size of the unspanned component of the SDFs, when $\mathbf{R}_{t+1}\left(\mathbf{R}_{t+1}^{*}\right)$ contains six assets

Reported are the $\Theta$ estimates, the SDF volatilities, the correlation between the SDFs, and the size of the unspanned components of the SDFs. There are 10 countries in our sample: Australia (AUD), New Zealand (NZD), United Kingdom (STG), France (FRA), Canada (CAD), United States (USD), Netherlands (NLG), Germany (GER), Japan (JPY), and Switzerland (SWI). The results are displayed in the order of decreasing average interest-rates (so Australia (AUD) has the highest average interest-rates, while Switzerland (SWI) has the lowest average interest-rates). The $95 \%$ lower and upper bootstrap confidence intervals are in square brackets.

\begin{tabular}{|c|c|c|c|c|c|c|c|}
\hline & \multicolumn{2}{|c|}{ Panel $A$} & \multicolumn{5}{|c|}{ Panel B: Properties of the domestic and foreign SDFs } \\
\hline & \multicolumn{2}{|c|}{ Bootstrap } & \multirow[b]{2}{*}{$\sigma[m]$} & \multirow[b]{2}{*}{$\sigma\left[m^{*}\right]$} & \multirow[b]{2}{*}{$\rho_{m, m^{*}}$} & \multirow[b]{2}{*}{$\frac{\operatorname{Var}[u]}{\operatorname{Var}[m]}$} & \multirow[b]{2}{*}{$\frac{\operatorname{Var}\left[u^{*}\right]}{\operatorname{Var}\left[m^{*}\right]}$} \\
\hline & $\Theta$ & $95 \%$ CI & & & & & \\
\hline AUD/NZD & 0.50 & {$\left[\begin{array}{ll}0.32 & 0.75\end{array}\right]$} & 42 & 43 & 0.26 & 0.39 & 0.33 \\
\hline AUD/STG & 0.73 & {$\left[\begin{array}{ll}0.48 & 0.91\end{array}\right]$} & 56 & 61 & 0.20 & 0.38 & 0.40 \\
\hline AUD/FRA & 0.63 & {$\left[\begin{array}{ll}0.42 & 0.84\end{array}\right]$} & 51 & 55 & 0.24 & 0.37 & 0.36 \\
\hline $\mathrm{AUD} / \mathrm{CAD}$ & 0.50 & {$\left[\begin{array}{ll}0.32 & 0.71\end{array}\right]$} & 46 & 49 & 0.42 & 0.29 & 0.27 \\
\hline AUD/USD & 0.71 & {$\left[\begin{array}{ll}0.45 & 0.90\end{array}\right]$} & 59 & 64 & 0.30 & 0.35 & 0.33 \\
\hline AUD/NLG & 0.97 & [0.69 1.15] & 69 & 74 & 0.05 & 0.47 & 0.46 \\
\hline AUD/GER & 0.96 & {$\left[\begin{array}{ll}0.71 & 1.18\end{array}\right]$} & 63 & 68 & -0.11 & 0.56 & 0.52 \\
\hline AUD/JPY & 0.96 & {$\left[\begin{array}{ll}0.79 & 1.21\end{array}\right]$} & 61 & 67 & -0.19 & 0.59 & 0.55 \\
\hline AUD/SWI & 0.84 & {$\left[\begin{array}{lll}0.59 & 1.03\end{array}\right]$} & 63 & 69 & 0.14 & 0.49 & 0.43 \\
\hline NZD/STG & 0.45 & {$\left[\begin{array}{ll}0.23 & 0.65\end{array}\right]$} & 48 & 51 & 0.55 & 0.21 & 0.21 \\
\hline NZD/FRA & 0.73 & {$\left[\begin{array}{ll}0.52 & 0.97\end{array}\right]$} & 48 & 53 & -0.09 & 0.58 & 0.53 \\
\hline NZD/CAD & 0.49 & {$\left[\begin{array}{ll}0.26 & 0.73\end{array}\right]$} & 44 & 51 & 0.40 & 0.29 & 0.28 \\
\hline NZD/USD & 0.61 & {$\left[\begin{array}{ll}0.33 & 0.80\end{array}\right]$} & 51 & 58 & 0.32 & 0.34 & 0.32 \\
\hline NZD/NLG & 1.00 & {$\left[\begin{array}{ll}0.71 & 1.18\end{array}\right]$} & 67 & 72 & -0.06 & 0.59 & 0.58 \\
\hline NZD/GER & 0.98 & {$\left[\begin{array}{ll}0.80 & 1.24\end{array}\right]$} & 59 & 66 & -0.28 & 0.65 & 0.60 \\
\hline NZD/JPY & 0.85 & {$\left[\begin{array}{ll}0.68 & 1.20\end{array}\right]$} & 54 & 60 & -0.16 & 0.59 & 0.53 \\
\hline NZD/SWI & 1.13 & {$\left[\begin{array}{ll}0.83 & 1.33\end{array}\right]$} & 73 & 78 & -0.15 & 0.57 & 0.56 \\
\hline STG/FRA & 0.62 & {$\left[\begin{array}{ll}0.42 & 0.80\end{array}\right]$} & 54 & 55 & 0.35 & 0.31 & 0.33 \\
\hline $\mathrm{STG} / \mathrm{CAD}$ & 0.46 & {$\left[\begin{array}{ll}0.21 & 0.65\end{array}\right]$} & 49 & 50 & 0.54 & 0.26 & 0.22 \\
\hline STG/USD & 0.47 & {$\left[\begin{array}{ll}0.22 & 0.63\end{array}\right]$} & 52 & 55 & 0.61 & 0.23 & 0.18 \\
\hline STG/NLG & 0.76 & {$\left[\begin{array}{ll}0.47 & 0.92\end{array}\right]$} & 64 & 67 & 0.32 & 0.33 & 0.34 \\
\hline STG/GER & 0.72 & {$\left[\begin{array}{ll}0.47 & 0.91\end{array}\right]$} & 56 & 59 & 0.20 & 0.45 & 0.41 \\
\hline STG/JPY & 0.57 & {$\left[\begin{array}{ll}0.34 & 0.77\end{array}\right]$} & 52 & 56 & 0.36 & 0.31 & 0.30 \\
\hline STG/SWI & 0.97 & {$\left[\begin{array}{ll}0.72 & 1.14\end{array}\right]$} & 71 & 75 & 0.10 & 0.44 & 0.44 \\
\hline FRA/CAD & 0.65 & {$\left[\begin{array}{ll}0.43 & 0.85\end{array}\right]$} & 54 & 56 & 0.26 & 0.37 & 0.35 \\
\hline FRA/USD & 0.56 & {$\left[\begin{array}{ll}0.32 & 0.74\end{array}\right]$} & 53 & 56 & 0.45 & 0.28 & 0.25 \\
\hline FRA/NLG & 0.71 & {$\left[\begin{array}{ll}0.47 & 0.86\end{array}\right]$} & 63 & 65 & 0.39 & 0.30 & 0.30 \\
\hline FRA/GER & 0.57 & {$\left[\begin{array}{ll}0.27 & 0.75\end{array}\right]$} & 54 & 56 & 0.46 & 0.27 & 0.26 \\
\hline FRA/JPY & 0.55 & {$\left[\begin{array}{ll}0.38 & 0.78\end{array}\right]$} & 48 & 51 & 0.33 & 0.32 & 0.31 \\
\hline FRA/SWI & 0.56 & {$\left[\begin{array}{ll}0.31 & 0.73\end{array}\right]$} & 58 & 60 & 0.53 & 0.23 & 0.22 \\
\hline CAD/USD & 0.39 & {$\left[\begin{array}{ll}0.14 & 0.57\end{array}\right]$} & 47 & 49 & 0.66 & 0.21 & 0.18 \\
\hline $\mathrm{CAD} / \mathrm{NLG}$ & 0.68 & {$\left[\begin{array}{ll}0.42 & 0.86\end{array}\right]$} & 60 & 61 & 0.34 & 0.34 & 0.31 \\
\hline CAD/GER & 0.71 & {$\left[\begin{array}{ll}0.47 & 0.94\end{array}\right]$} & 57 & 59 & 0.22 & 0.40 & 0.36 \\
\hline CAD/JPY & 0.94 & {$\left[\begin{array}{ll}0.66 & 1.16\end{array}\right]$} & 64 & 67 & -0.06 & 0.52 & 0.52 \\
\hline CAD/SWI & 0.67 & {$\left[\begin{array}{ll}0.35 & 0.85\end{array}\right]$} & 59 & 61 & 0.36 & 0.44 & 0.37 \\
\hline USD/NLG & 0.64 & {$\left[\begin{array}{ll}0.40 & 0.80\end{array}\right]$} & 64 & 64 & 0.49 & 0.25 & 0.25 \\
\hline USD/GER & 0.56 & {$\left[\begin{array}{ll}0.35 & 0.75\end{array}\right]$} & 53 & 53 & 0.42 & 0.29 & 0.30 \\
\hline USD/JPY & 0.73 & {$\left[\begin{array}{ll}0.48 & 0.91\end{array}\right]$} & 59 & 61 & 0.24 & 0.37 & 0.38 \\
\hline USD/SWI & 0.80 & {$\left[\begin{array}{ll}0.51 & 0.97\end{array}\right]$} & 65 & 66 & 0.24 & 0.38 & 0.37 \\
\hline NLG/GER & 0.52 & {$\left[\begin{array}{ll}0.31 & 0.67\end{array}\right]$} & 57 & 57 & 0.59 & 0.23 & 0.22 \\
\hline NLG/JPY & 0.87 & [0.62 1.05] & 67 & 69 & 0.15 & 0.41 & 0.42 \\
\hline NLG/SWI & 0.76 & {$\left[\begin{array}{ll}0.48 & 0.92\end{array}\right]$} & 67 & 69 & 0.36 & 0.31 & 0.32 \\
\hline GER/JPY & 0.47 & {$\left[\begin{array}{ll}0.28 & 0.71\end{array}\right]$} & 47 & 48 & 0.47 & 0.24 & 0.25 \\
\hline GER/SWI & 0.59 & {$\left[\begin{array}{ll}0.30 & 0.76\end{array}\right]$} & 59 & 60 & 0.51 & 0.24 & 0.25 \\
\hline JPY/SWI & 0.99 & {$\left[\begin{array}{ll}0.71 & 1.17\end{array}\right]$} & 72 & 72 & 0.03 & 0.49 & 0.46 \\
\hline
\end{tabular}


Table 3: A snapshot of SDF volatilities, correlation between SDFs, and the size of the unspanned components

Reported is a snapshot of (i) SDF volatilities $\sigma[m]$ and $\sigma\left[m^{*}\right]$, (ii) correlation between SDFs $\rho_{m, m^{*}}$, and (iii) the size of the unspanned components of the SDFs $\frac{\operatorname{Var}[u]}{\operatorname{Var}[m]}$ and $\frac{\operatorname{Var}\left[u^{*}\right]}{\operatorname{Var}\left[m^{*}\right]}$. $\mathbf{R}_{t+1}$ with six assets, in the case of AUD/JPY, contains the returns of (i) the Australian risk-free bond, (ii) the Australian equity index, (iii) the Japanese risk-free bond, (iv) the Japanese equity index, (v) the U.S. 30-year treasury bond, (vi) the MSCI world index, where each of the returns are denominated in Australian dollars, while $\mathbf{R}_{t+1}^{*}$ contains the same set of assets denominated in Japanese yen. The $\mathbf{R}_{t+1}\left(\mathbf{R}_{t+1}^{*}\right)$ with four assets is a restricted counterpart of that with six assets, with the returns of (i) the Australian risk-free bond, (ii) the Australian equity index, (iii) the Japanese risk-free bond, and (iv) the Japanese equity index.

Mean SD Min. Max. $\quad$\begin{tabular}{c} 
Percentiles \\
\cline { 3 - 5 }
\end{tabular}

\begin{tabular}{|c|c|c|c|c|c|c|c|c|c|c|}
\hline \multicolumn{11}{|c|}{ Panel A: SDF volatilities } \\
\hline \multirow[t]{2}{*}{$\sigma[m]$} & Six assets & 57 & 8 & 42 & 73 & 46 & 52 & 57 & 63 & 71 \\
\hline & Four assets & 54 & 8 & 38 & 70 & 41 & 48 & 53 & 60 & 67 \\
\hline \multirow[t]{2}{*}{$\sigma\left[m^{*}\right]$} & Six assets & 60 & 8 & 43 & 78 & 49 & 55 & 60 & 67 & 74 \\
\hline & Four assets & 57 & 9 & 42 & 77 & 43 & 51 & 57 & 63 & 71 \\
\hline \multicolumn{11}{|c|}{ Panel B: Correlation between SDFs } \\
\hline \multirow[t]{2}{*}{$\rho_{m, m^{*}}$} & Six assets & 0.26 & 0.24 & -0.28 & 0.66 & -0.16 & 0.14 & 0.32 & 0.42 & 0.58 \\
\hline & Four assets & 0.26 & 0.24 & -0.28 & 0.66 & -0.16 & 0.14 & 0.32 & 0.42 & 0.58 \\
\hline \multicolumn{11}{|c|}{ Panel C: Size of the unspanned component of the SDFs } \\
\hline \multirow{2}{*}{$\frac{\operatorname{Var}[u]}{\operatorname{Var}[m]}$} & Six assets & 0.38 & 0.12 & 0.21 & 0.65 & 0.23 & 0.29 & 0.35 & 0.45 & 0.59 \\
\hline & Four assets & 0.36 & 0.12 & 0.17 & 0.64 & 0.21 & 0.27 & 0.33 & 0.43 & 0.58 \\
\hline \multirow{2}{*}{$\frac{\operatorname{Var}\left[u^{*}\right]}{\operatorname{Var}\left[m^{*}\right]}$} & Six assets & 0.36 & 0.11 & 0.18 & 0.60 & 0.21 & 0.27 & 0.33 & 0.43 & 0.56 \\
\hline & Four assets & 0.35 & 0.11 & 0.16 & 0.60 & 0.21 & 0.26 & 0.33 & 0.43 & 0.53 \\
\hline
\end{tabular}


Table 4: Regressions of (log) real exchange rate growth on (log) relative SDFs

The U.S. (USD), Japan (JPY), or Switzerland (SWI) is the domestic country versus all other remaining foreign countries. Reported are the results for the following time-series regression:

$$
\log \left(\frac{S_{t+1}}{S_{t}}\right)=\Pi_{0}+\Pi_{1} \log \left(\frac{m_{t+1}^{*}}{m_{t+1}}\right)+e_{t+1}
$$

where $S_{t}$ is the exchange rate (the domestic currency price per 1 unit of the foreign currency) and $e_{t+1}$ is the regression disturbance. Complete markets imply $\Pi_{0}=0$ and $\Pi_{1}=1$. The SDF pairs $\left(m_{t+1}, m_{t+1}^{*}\right)$ are synthesized from Problem 1. We report the coefficient estimates and the corresponding $t$-statistics. The $t$-statistics are based on the procedure in Newey and West (1987) with optimal lag selected as in Newey and West (1994), and the adjusted $R^{2}$ (in \%) is denoted by $\bar{R}^{2}$. We perform the Wald test for the hypothesis $\Pi_{1}=1$ and report the $p$-values in square brackets. CORR is the correlation between $\log \left(\frac{S_{t+1}}{S_{t}}\right)$ and $\log \left(\frac{m_{t+1}^{*}}{m_{t+1}}\right)$. The sample period is January 1975 to June 2014 (474 observations).

\begin{tabular}{|c|c|c|c|c|c|c|c|c|c|c|c|}
\hline \multirow[b]{2}{*}{ Domestic } & & \multicolumn{10}{|c|}{ Foreign currency is the reference against the domestic } \\
\hline & & AUD & NZD & STG & FRA & $\mathrm{CAD}$ & USD & NLG & GER & JPY & SWI \\
\hline \multirow[t]{5}{*}{ USD } & $\begin{array}{c}\Pi_{0} \\
(t \text {-stat })\end{array}$ & $\begin{array}{c}0.00 \\
(0.11)\end{array}$ & $\begin{array}{c}0.00 \\
(0.42)\end{array}$ & $\begin{array}{c}0.00 \\
(0.19)\end{array}$ & $\begin{array}{c}-0.00 \\
(-0.06)\end{array}$ & $\begin{array}{c}-0.00 \\
(-0.16)\end{array}$ & - & $\begin{array}{c}0.00 \\
(-0.05)\end{array}$ & $\begin{array}{c}-0.00 \\
(-0.09)\end{array}$ & $\begin{array}{c}0.00 \\
(0.28)\end{array}$ & $\begin{array}{c}0.00 \\
(0.34)\end{array}$ \\
\hline & $\underset{(t \text {-stat })}{\Pi_{1}}$ & $\begin{array}{c}0.03 \\
(3.41)\end{array}$ & $\begin{array}{c}0.03 \\
(3.16)\end{array}$ & $\begin{array}{c}0.03 \\
(1.85)\end{array}$ & $\begin{array}{c}0.07 \\
(5.60)\end{array}$ & $\begin{array}{c}0.02 \\
(2.55)\end{array}$ & & $\begin{array}{c}0.02 \\
(2.76)\end{array}$ & $\begin{array}{c}0.03 \\
(2.78)\end{array}$ & $\begin{array}{c}0.02 \\
(2.02)\end{array}$ & $\begin{array}{c}0.02 \\
(2.52)\end{array}$ \\
\hline & $\bar{R}^{2}(\%)$ & 3.0 & 2.7 & 1.1 & 3.1 & 1.3 & & 1.6 & 1.7 & 1.00 & 1.6 \\
\hline & $\begin{array}{l}\Pi_{1}=1 \\
{[p \text {-val. }]}\end{array}$ & {$[0.00]$} & {$[0.00]$} & {$[0.00]$} & {$[0.00]$} & {$[0.00]$} & & {$[0.00]$} & {$[0.00]$} & {$[0.00]$} & {$[0.00]$} \\
\hline & CORR & 0.18 & 0.17 & 0.11 & 0.19 & 0.12 & & 0.14 & 0.14 & 0.11 & 0.13 \\
\hline \multirow[t]{5}{*}{ JPY } & $\begin{array}{c}\Pi_{0} \\
(t \text {-stat })\end{array}$ & $\begin{array}{c}-0.00 \\
(-0.09)\end{array}$ & $\begin{array}{c}0.00 \\
(0.16)\end{array}$ & $\begin{array}{c}-0.00 \\
(-0.09)\end{array}$ & $\begin{array}{c}-0.00 \\
(-0.27)\end{array}$ & $\begin{array}{c}-0.00 \\
(-0.29)\end{array}$ & $\begin{array}{c}-0.00 \\
(-0.25)\end{array}$ & $\begin{array}{c}-0.00 \\
(-0.20)\end{array}$ & $\begin{array}{c}-0.00 \\
(-0.30)\end{array}$ & - & $\begin{array}{c}0.00 \\
(0.08)\end{array}$ \\
\hline & $\begin{array}{c}\Pi_{1} \\
(t \text {-stat })\end{array}$ & $\begin{array}{c}0.04 \\
(4.73)\end{array}$ & $\begin{array}{c}0.06 \\
(5.18)\end{array}$ & $\begin{array}{c}0.04 \\
(3.33)\end{array}$ & $\begin{array}{c}0.07 \\
(5.60)\end{array}$ & $\begin{array}{c}0.03 \\
(4.36)\end{array}$ & $\begin{array}{c}0.03 \\
(4.09)\end{array}$ & $\begin{array}{c}0.02 \\
(4.06)\end{array}$ & $\begin{array}{c}0.08 \\
(6.11)\end{array}$ & & $\begin{array}{c}0.02 \\
(3.43)\end{array}$ \\
\hline & $\bar{R}^{2}(\%)$ & 4.5 & 6.9 & 3.0 & 7.7 & 3.6 & 4.4 & 2.0 & 7.9 & & 2.1 \\
\hline & $\begin{array}{l}\Pi_{1}=1 \\
{[p \text {-val. }]}\end{array}$ & {$[0.00]$} & {$[0.00]$} & {$[0.00]$} & {$[0.00]$} & {$[0.00]$} & {$[0.00]$} & {$[0.00]$} & {$[0.00]$} & & {$[0.00]$} \\
\hline & CORR & 0.22 & 0.27 & 0.18 & 0.28 & 0.20 & 0.21 & 0.15 & 0.28 & & 0.15 \\
\hline \multirow[t]{5}{*}{ SWI } & $\begin{array}{c}\Pi_{0} \\
(t \text {-stat })\end{array}$ & $\begin{array}{c}-0.00 \\
(-0.18)\end{array}$ & $\begin{array}{c}0.00 \\
(0.13)\end{array}$ & $\begin{array}{c}-0.00 \\
(-0.21)\end{array}$ & $\begin{array}{l}-0.00 \\
(-0.80)\end{array}$ & $\begin{array}{c}-0.00 \\
(-0.38)\end{array}$ & $\begin{array}{l}-0.00 \\
-0.33\end{array}$ & $\begin{array}{c}-0.00 \\
(-0.70)\end{array}$ & $\begin{array}{c}-0.00 \\
(-0.99)\end{array}$ & $\begin{array}{c}-0.00 \\
(-0.06)\end{array}$ & - \\
\hline & $\begin{array}{c}\Pi_{1} \\
(t \text {-stat })\end{array}$ & $\begin{array}{c}0.02 \\
(3.33)\end{array}$ & $\begin{array}{c}0.01 \\
(1.71)\end{array}$ & $\begin{array}{c}0.00 \\
(0.22)\end{array}$ & $\begin{array}{c}0.01 \\
(2.23)\end{array}$ & $\begin{array}{c}0.03 \\
(3.37)\end{array}$ & $\begin{array}{c}0.02 \\
(2.97)\end{array}$ & $\begin{array}{l}-0.00 \\
(-0.06)\end{array}$ & $\begin{array}{c}0.00 \\
(0.05)\end{array}$ & $\begin{array}{c}0.01 \\
(1.87)\end{array}$ & \\
\hline & $\bar{R}^{2}(\%)$ & 2.6 & 0.6 & 0.0 & 1.1 & 2.4 & 2.2 & 0.0 & 0.0 & 0.50 & \\
\hline & $\begin{array}{l}\Pi_{1}=1 \\
{[p \text {-val. }]}\end{array}$ & {$[0.00]$} & {$[0.00]$} & {$[0.00]$} & {$[0.00]$} & {$[0.00]$} & {$[0.00]$} & {$[0.00]$} & {$[0.00]$} & {$[0.00]$} & \\
\hline & CORR & 0.17 & 0.09 & 0.01 & 0.11 & 0.16 & 0.15 & -0.00 & 0.00 & 0.08 & \\
\hline
\end{tabular}




\title{
Studying the Implications of Consumption and Asset Return Data for Stochastic Discount Factors in Incomplete International Economies
}

\author{
Gurdip Bakshi Mario Cerrato John Crosby
}

\section{Internet Appendix: Not for Publication}

\begin{abstract}
This internet appendix provides additional theoretical elaboration and data documentation.

The thrust of Section I is an example economy in which $m\left(S_{t+1} / S_{t}\right)-m^{\star}$ need not be zero in each state of the world, and the domestic country has low interest-rates, while the foreign country has high interest-rates. We describe the parameterized economy with five states and present the results on the properties of $\left(m, m^{*}\right)$ in Table Internet-I.

Section II outlines the data sources.

Table Internet-II illustrates the properties of $\left(m, m^{*}\right)$ pairs when $\mathbf{R}_{t+1}\left(\mathbf{R}_{t+1}^{*}\right)$ contains seven assets. Finally, Table Internet-III displays the results of a robustness exercise when the $\Theta$ estimate is constrained to be the same across all industrialized countries.
\end{abstract}




\section{An incomplete markets model parameterized by five states}

In Table Internet-I, we present a particularly parameterized two-country economy with five states of the world, featuring that $m\left(S_{t+1} / S_{t}\right)-m^{\star}$ need not be zero in each state.

The economy is constructed to capture some relevant features. First, the domestic country supports a low risk-free interest-rate (say, Japan), whereas the foreign country (say, Australia) a high risk-free interest-rate. Second, the returns of the risky asset (i.e., equity) display positive correlation.

Our objective is to illustrate the solution technique and highlight the volatilities and correlation between $m$ and $m^{\star}$. We further show that the problem is well-posed with a finite objective and well-defined Lagrange multipliers, and the solution supports $m>0$ and $m^{\star}>0$ over a wide range of values of $\Theta$.

We compute $e_{\mathbf{z}}$ by solving equation (40) and then $d$ and $d^{\star}$, as described in equation (41). The solution method for computing $e_{\mathbf{z}}$ is to regress one on $\mathbf{Z}$. Armed with $y_{\mathbf{z}}, \delta, d$, and $d^{\star}$, we compute $y$ and $y^{\star}$ using equation (33). We verify our solution and check if $\mathbb{E}[\delta \mathbf{Z}]=0$, for each element of $\mathbf{Z}$. As in Subsection 2.3 , we verify that $\mathbb{E}\left[y-y^{\star}\right]$ is not far from zero.

With the computed values of $y$ and $y^{\star}$, we obtain $m$ and $m^{\star}$ across the five states using equation (10). Prompted by the specifics of our solution, we compute (i) $\sqrt{\operatorname{Var}[m]}$ and $\sqrt{\operatorname{Var}\left[m^{\star}\right]}$, and (ii) the correlation $\rho_{m, m^{*}}$.

The question is: If the market incompleteness parameter $\Theta$ were to be assumed close to zero,

how would the properties of $\left(m, m^{*}\right)$ change by ruling out "good deals," that is, $\mathbb{E}\left[\left(y-y^{\star}\right)^{2}\right] \leq \Theta^{2}$ in comparison with $\mathbb{E}\left[\left(y-y^{\star}\right)^{2}\right] \approx 0$. As seen, the economy supports a lower correlation $\rho_{m, m^{*}}$ but with the added effect of raising the volatility of $m$ and $m^{\star}$.

We also check our solution by directly minimizing the objective in (29), subject to the constraints in $(30)-(32)$.

In summary, we construct a discrete-time, five-state economy in which $m\left(S_{t+1} / S_{t}\right)-m^{\star}$ need not be zero and the domestic and foreign country pairs have low and high risk-free interest-rates, respectively. We show that the nonnegativity constraints do not bind, and $m$ and $m^{\star}$ are strictly 
positive in each state. Crucially, increasing the market incompleteness parameter reduces the correlation between $m$ and $m^{\star}$, while increasing the volatility of $m$ and $m^{\star}$.

\section{Data description and sources}

The nominal returns of bonds, equity indexes, and currencies are converted into real returns by adjusting by ex-post realized inflation. The sources of the data are described below:

Interest rates and bonds: Risk-free bond prices are constructed from LIBOR quotes as $1 /(1+$ $\tau$ LIBOR), where $\tau$ is the day count fraction, that is, $\tau=1 / 12$ for monthly. When LIBOR is not available, we use the nearest substitute, such as 30-day Bank Bill rates (which are money market rates). At the start of the historical time period considered, interest-rate data for JPY, AUD, and NZD was not available from Datastream, so we collect data from the respective central banks.

The data on the returns of 30-year U.S. Treasury bond is from WRDS.

Equity returns: The equity index return data (including that for the world equity index) is MSCI data from Datastream, and we employ total returns (including dividends). MSCI data is not available for New Zealand prior to 1988, so for the period 1975-1987, we use returns data supplied by Martin Lally and Alastair Marsden.

Exchange rates: The spot exchange rate data for all country pairs is the midpoint of the bid and ask quotes (from Datastream). The exchange rates for France, Germany, and Netherlands from January 1999 (the introduction of the Euro) onward are taken to be the relevant fixed conversion rate to the Euro (e.g., DM $1.95583=1$ Euro).

Inflation: Country-specific inflation data is from Datastream and is CPI data. For the United Kingdom and France, we splice the CPI data with retail price index data for the periods 1975 to 1988 and 1975 to 1989 , respectively.

Consumption: We use annual real consumption growth data from Barro and Ursua (2008), updated using World Development Indicators.

Commodities: S\&P commodity index data is from Datastream (ticker: OFCL). 
Table Internet-I: Properties of $m$ and $m^{*}$ in an example economy with five states and where $m\left(S_{t+1} / S_{t}\right)-m^{\star} \neq 0$

In our illustrative calculations, the domestic country is Japan (with a low risk-free interest-rate), and the foreign country is Australia (with a high risk-free interest-rate). The exchange rate growth $S_{t+1} / S_{t}$ is denominated in $¥ \mid \mathrm{AD}$. We follow the steps in Subsection 2.5 and compute $e_{\mathbf{z}}$ by solving equation (40) and then $d$ and $d^{\star}$ via equation (41). The computer code for obtaining the solution by minimizing the objective in (29), subject to the constraints in equations (30)-(32), is available from the authors (in $\mathrm{C}++$ and in an Excel spreadsheet setting). $\sigma[m]$ and $\sigma\left[m^{*}\right]$ are the volatilities of $m$ and $m^{*}$, and $\rho_{m, m^{*}}$ is the correlation between $m$ and $m^{*}$.

\section{Panel A: Parametrization of the economy}

States of the world

$j=1 \quad j=2 \quad j=3 \quad j=4 \quad j=5$

\begin{tabular}{llllll}
\hline & & & & & \\
Probability & 0.07 & 0.20 & 0.45 & 0.21 & 0.07 \\
Risk-free (domestic) & 1.01 & 1.01 & 1.01 & 1.01 & 1.01 \\
Risk-free (foreign) & 1.04 & 1.04 & 1.04 & 1.04 & 1.04 \\
Risky (domestic) & 0.87 & 1.01 & 1.04 & 1.05 & 1.08 \\
Risky (foreign) & 0.87 & 1.01 & 1.08 & 1.04 & 1.42 \\
Exchange rate growth & 0.90 & 0.89 & 1.00 & 1.11 & 1.10 \\
& & & & & \\
\hline
\end{tabular}

Panel B: Properties of $m$ and $m^{*}$ obtained by varying $\Theta$

\begin{tabular}{|c|c|c|c|c|c|c|c|c|}
\hline & \multicolumn{5}{|c|}{ States of the world } & \multirow[b]{2}{*}{ Mean } & \multirow[b]{2}{*}{$\begin{array}{c}\sigma[m] \\
\left(\sigma\left[m^{*}\right]\right)\end{array}$} & \multirow[b]{2}{*}{$\rho_{m, m^{*}}$} \\
\hline & $j=1$ & $j=2$ & $j=3$ & $j=4$ & $j=5$ & & & \\
\hline \multicolumn{9}{|l|}{$\Theta=0.05$} \\
\hline$m$ & 1.9887 & 1.3949 & 0.9410 & 0.5724 & 0.4036 & 0.9901 & 41 & \\
\hline \multirow[t]{2}{*}{$m^{\star}$} & 1.7763 & 1.2975 & 0.8889 & 0.6841 & 0.4858 & 0.9615 & $(32)$ & \\
\hline & & & & & & & & 0.99 \\
\hline \multicolumn{9}{|l|}{$\Theta=0.40$} \\
\hline$m$ & 2.0415 & 1.1745 & 1.1232 & 0.4188 & 0.2706 & 0.9901 & 44 & \\
\hline \multirow[t]{2}{*}{$m^{\star}$} & 1.7288 & 1.4937 & 0.7068 & 0.8547 & 0.6321 & 0.9615 & $(37)$ & \\
\hline & & & & & & & & 0.55 \\
\hline \multicolumn{9}{|l|}{$\Theta=0.60$} \\
\hline$m$ & 2.0716 & 1.0485 & 1.2273 & 0.3309 & 0.1946 & 0.9901 & 49 & \\
\hline \multirow[t]{2}{*}{$m^{\star}$} & 1.7017 & 1.6058 & 0.6027 & 0.9522 & 0.7157 & 0.9615 & $(43)$ & \\
\hline & & & & & & & & 0.19 \\
\hline \multicolumn{9}{|l|}{$\Theta=0.80$} \\
\hline$m$ & 2.1017 & 0.9225 & 1.3314 & 0.2431 & 0.1186 & 0.9901 & 56 & \\
\hline \multirow[t]{2}{*}{$m^{\star}$} & 1.6746 & 1.7179 & 0.4986 & 1.0496 & 0.7993 & 0.9615 & $(50)$ & \\
\hline & & & & & & & & -0.11 \\
\hline
\end{tabular}


Table Internet-II: A snapshot of $\Theta$ estimates, SDF volatilities, correlation between SDFs, and the size of the unspanned components when $\mathbf{R}_{t+1}\left(\mathbf{R}_{t+1}^{*}\right)$ contains seven assets

Estimation algorithm incorporates the argument that there exists a value of $\Theta$ that minimizes the discrepancy between the risk sharing index from consumption and asset returns (as detailed in Section 3.1.1). We focus on a set of asset returns that allows for multi-currency, multi-country, and commodity index exposures. Consider the Australia versus Japan (AUD/JPY) country pair, in which the the gross return vector $\mathbf{R}_{t+1}$ contains seven assets:

$$
\underbrace{\mathbf{R}_{t+1}}_{7 \times 1}=\left\{\begin{array}{l}
\text { Return of the Australian risk-free bond (in AUD) } \\
\text { Return of the Australian equity index (in AUD) } \\
\text { Return of the Japanese risk-free bond (in AUD) } \\
\text { Return of the Japanese equity index (in AUD) } \\
\text { Return of the U.S. 30-year Treasury bond (in AUD) } \\
\text { Return of the MSCI world equity index (in AUD) } \\
\text { Return of the S\&P commodity index (in AUD) }
\end{array}\right.
$$

Symmetrically, $\mathbf{R}_{t+1}^{*}=\mathbf{R}_{t+1} /\left(\frac{S_{t+1}}{S_{t}}\right)$ contains the same set of gross returns denominated in Japanese yen, where $\frac{S_{t+1}}{S_{t}}$ is the exchange rate growth with the Japanese yen as the reference currency. Reported is a snapshot of (i) $\Theta$ estimates, (ii) SDF volatilities, (iii) correlation between SDFs, and (iv) the unspanned components of the SDFs. The sample period considered is January 1975 to June 2014 (474 observations).

Mean SD Min. Max. $\quad$\begin{tabular}{c} 
Percentiles \\
\cline { 3 - 5 }
\end{tabular}

\section{Panel A: $\Theta$ estimates}

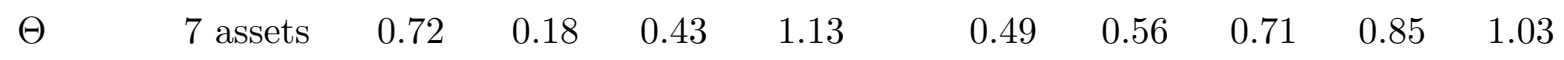

Panel B: SDF volatilities

$\begin{array}{lllllllllll}\sigma[m] & 7 \text { assets } & 59 & 7 & 48 & 73 & 48 & 53 & 58 & 64 & 71\end{array}$

$\begin{array}{lllllllllll}\sigma\left[m^{*}\right] & 7 \text { assets } & 62 & 8 & 49 & 78 & 51 & 56 & 60 & 67 & 75\end{array}$

Panel C: Correlation between SDFs

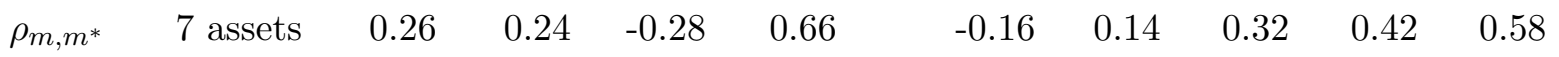

Panel D: Size of the unspanned component of the SDFs

$\begin{array}{ccccccccccc}\frac{\operatorname{Var}[u]}{\operatorname{Var}[m]} & 7 \text { assets } & 0.40 & 0.13 & 0.21 & 0.67 & 0.23 & 0.29 & 0.37 & 0.47 & 0.65 \\ \frac{\operatorname{Var}\left[u^{*}\right]}{\operatorname{Var}\left[m^{*}\right]} & 7 \text { assets } & 0.37 & 0.12 & 0.18 & 0.62 & 0.21 & 0.30 & 0.35 & 0.44 & 0.59\end{array}$


Table Internet-III: A snapshot of SDF volatilities, correlation between SDFs, and the size of the unspanned components when $\Theta$ is constrained to 0.65 across all industrialized countries

Reported is a snapshot of SDF volatilities $\sigma[m]$ and $\sigma\left[m^{*}\right]$, correlation between SDFs $\rho_{m, m^{*}}$, and the size of the unspanned components of the SDFs $\frac{\operatorname{Var}[u]}{\operatorname{Var}[m]}$ and $\frac{\operatorname{Var}\left[u^{*}\right]}{\operatorname{Var}\left[m^{*}\right]} \cdot \mathbf{R}_{t+1}$ with six assets, in the case of AUD/JPY, contains the returns of (i) the Australian risk-free bond, (ii) the Australian equity index, (iii) the Japanese risk-free bond, (iv) the Japanese equity index, (v) the U.S. 30year Treasury bond, (vi) the MSCI world index, where each of the returns are denominated in Australian dollars, while $\mathbf{R}_{t+1}^{*}$ contains the same set of assets denominated in Japanese yen. The $\mathbf{R}_{t+1}\left(\mathbf{R}_{t+1}^{*}\right)$ with four assets is a restricted counterpart of that with six assets, with the returns of (i) the Australian risk-free bond, (ii) the Australian equity index, (iii) the Japanese risk-free bond, and (iv) the Japanese equity index. The sample period considered is January 1975 to June 2014 (474 observations).

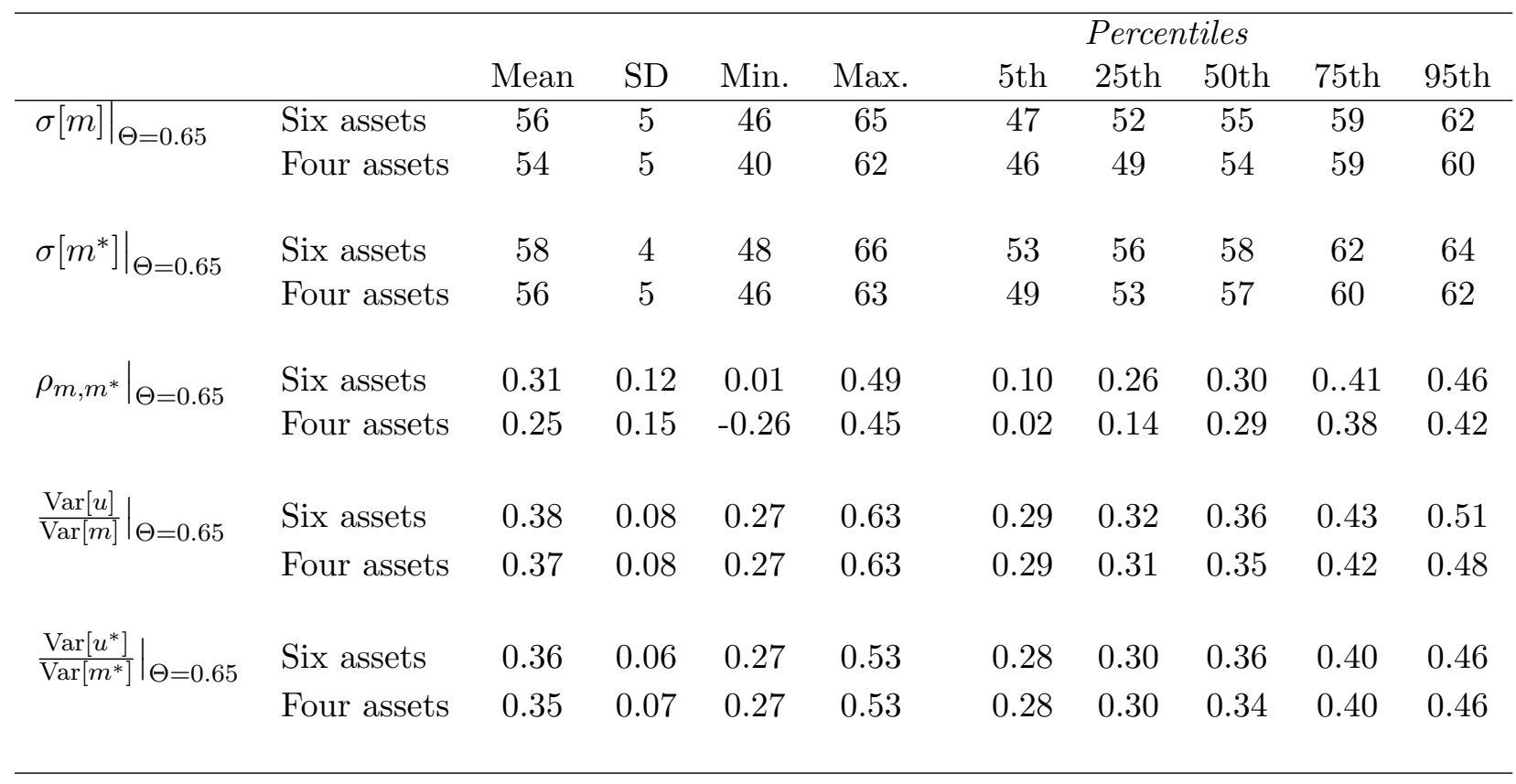

